\title{
Shallow viscoplastic flow on an inclined plane
}

\author{
By N. J. BALMFORTH ${ }^{1}$, R. V. CRASTER ${ }^{2}$ AND R. SASSI ${ }^{1}$ \\ ${ }^{1}$ Department of Applied Mathematics and Statistics, School of Engineering, \\ University of California at Santa Cruz, CA 95064, USA \\ ${ }^{2}$ Department of Mathematics, Imperial College of Science, Technology and Medicine, \\ London, SW7 2BZ, UK
}

(Received 13 December 2001 and in revised form 20 April 2002)

Evolving viscoplastic flows upon slopes are an important idealization of many flows in a variety of geophysical situations where yield stress is thought to play a role. For such models, asymptotic expansions suitable for slowly moving shallow fluid layers (lubrication theory) reduce the governing equations to a simpler problem in terms of the fluid thickness. We consider the version of the theory for fluids described by the Herschel-Bulkley constitutive law, and provide a variety of solutions to the reduced equation, both numerical and analytical. For extruded inclined domes, we derive the characteristic temporal behaviour of measures of the dome's dimensions.

\section{Introduction}

A key ingredient in many geophysical problems is the spreading of a layer of fluid under the combined action of gravity and internal stresses. Examples include the downhill flow of lava (Griffiths 2000), the movement of ice masses (Hutter 1983), and the release and slide of mud piles (Liu \& Mei 1989). Several researchers have modelled such spreading dynamics by treating the fluid as a shallow Newtonian fluid (Huppert 1982; Lister 1992). However, many geological materials are distinctly non-Newtonian in character: ice is commonly assumed to flow like a power-law fluid (Hutter 1983), and lava and muds are often modelled (both theoretically and in the laboratory) as shear-thinning viscoplastic fluids (Griffiths 2000; Coussot 1997). Avalanches and debris flows are sometimes modelled as viscoplastic fluids (e.g. Whipple 1997), though with some skepticism on the physical suitability.

Problems involving spreading fluids also arise in a variety of chemical engineering contexts, where non-Newtonian rheology is typically the standard, rather than the exception. Here, many industrial processes involve the flow of specially designed fluids in thin layers with the frequent aim of preparing uniform coatings. In fact, the shape adopted by viscoplastic fluids flowing on horizontal or inclined planes has even been suggested as a means to measure rheology (DeKee et al. 1990; Coussot, Proust \& Ancey 1996).

Of course, there are many complicating factors in both the geophysical and engineering applications. For example, the thermal structure of lava and ice is an important factor in the flow problem, primarily because slight temperature variations can generate relatively large changes in rheology. We ignore all such complicating factors in this study, and give a general discussion of the flow of an isothermal viscoplastic fluid on an inclined surface. The only approximations we make are that the fluid layer is thin and slowly moving, which allows us to take advantage of a lubrication-style 


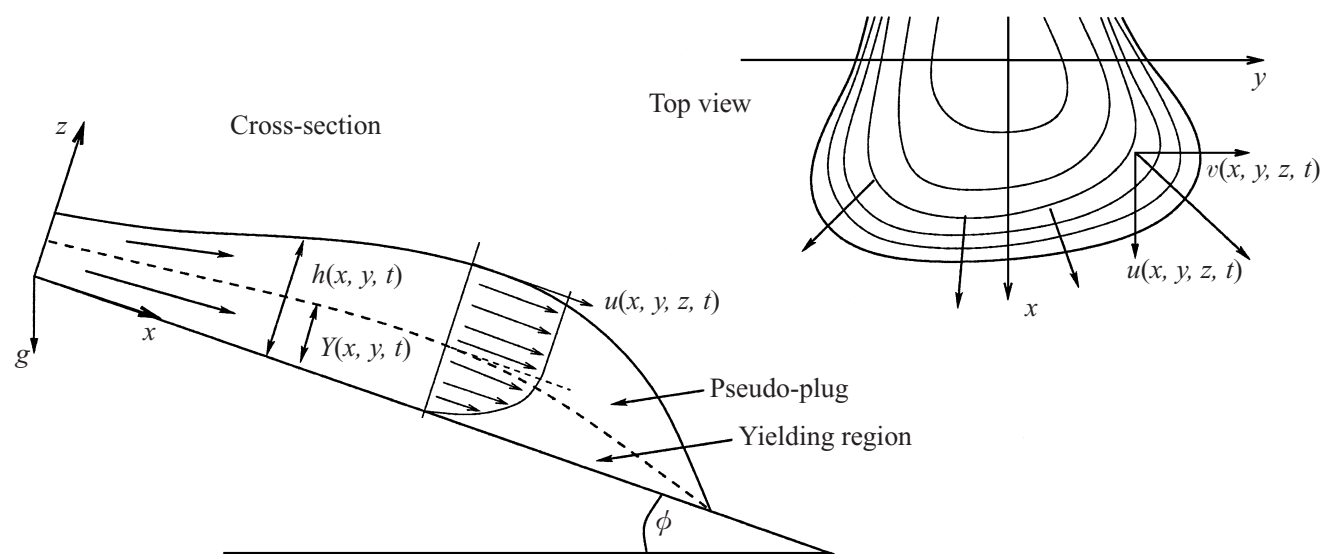

FIGURE 1. Sketch of a flow on an inclined plane. $\phi$ is the angle of inclination to the horizontal, $z$ is perpendicular to the plane, $x$ is directed downslope, and $y$ is the cross-slope coordinate.

asymptotic reduction of the governing fluid equations. This reduction scheme is standard in many fields for Newtonian fluids; here we adopt the version of this theory for a viscoplastic fluid based on the Herschel-Bulkley model (a non-Newtonian fluid model that incorporates both a yield stress and shear thinning or thickening). Our aim is provide a detailed exploration of this relatively unstudied problem, and to lay a foundation for future discussions of the particular applications in geophysics and engineering. Previous results are confined to a limited selection of solutions for static domes (Coussot et al. 1996; Osmond \& Griffiths 2001), steady three-dimensional flows (Coussot \& Proust 1996), and two-dimensional sheet flows (Liu \& Mei 1989; Huang \& Garcia 1998). Most recently, Mei \& Yuhi (2001) have presented a solution describing a dam-break problem for viscoplastic flow in a channel.

\section{The reduced model}

We consider the configuration shown in figure 1, described by a Cartesian coordinate system $(x, y, z) ; x$ is the downstream coordinate, $y$ denotes the cross-stream coordinate and $z=0$ locates the inclined plane. The angle of inclination of the plane to the horizontal is $\phi$. The thickness of the fluid is $h(x, y, t)$ and $(u, v)$ denotes the velocity field. There are three typical scenarios for the emplacement of the fluid: (i) the fluid is extruded onto the plane through a vent (Hulme 1974; Osmond \& Griffiths 2001); (ii) the fluid is fed onto the plane from an upstream reservoir (Coussot \& Proust 1996); (iii) a fixed volume of fluid is allowed to slump to equilibrium.

We describe the fluid by the Herschel-Bulkley constitutive law (e.g. Tanner 1985). In this non-Newtonian model, the components of the stresses, $\tau_{i j}$, are related to the strain rates, defined in terms of $\dot{\gamma}_{i j} \equiv \partial u_{i} / \partial x_{j}+\partial u_{j} / \partial x_{i}$, through the relations

$$
\tau_{i j}=\left(K \dot{\gamma}^{n-1}+\frac{\tau_{p}}{\dot{\gamma}}\right) \dot{\gamma}_{i j} \text { for } \tau \geqslant \tau_{p}
$$

and

$$
\dot{\gamma}_{i j}=0 \text { for } \tau<\tau_{p}
$$

where $\tau_{p}$ is the yield stress, $K$ is the 'consistency', $n$ is a power-law parameter, and the second invariants are

$$
\tau=\sqrt{\tau_{i j} \tau_{i j} / 2}, \quad \dot{\gamma}=\sqrt{\dot{\gamma}_{i j} \dot{\gamma}_{i j} / 2}
$$


With the constitutive law in hand, the next step is to specify the momentum and continuity equations for the fluid, and the boundary conditions on the base (no-slip) and surface (stress-free). From there, we pick a suitable non-dimensionalization of the equations in order to isolate a small parameter (namely $\epsilon=H / L$, the ratio of characteristic thickness, $H$, to characteristic scale, $L$, in $x$ and $y$ ) and determine the largest terms in each equation. The leading-order balances can then be manipulated into the required thin-layer equations. We do not give the details here, and refer the reader to Balmforth \& Craster (1999) and Mei \& Yuhi (2001). We quote only the final reduced model:

$$
h_{t}+\mathscr{U}_{x}+\mathscr{V}_{y}=w_{s}, \quad\left(\begin{array}{c}
\mathscr{U} \\
\mathscr{V}
\end{array}\right)=\frac{n Y(s Y)^{1 / n}[(1+2 n) h-n Y]}{s(n+1)(2 n+1)}\left(\begin{array}{c}
S-h_{x} \\
-h_{y}
\end{array}\right),
$$

with

$$
Y(x, y, t)=\operatorname{Max}\left(h-\frac{B}{S}, 0\right) \quad \text { and } s=\sqrt{\left(S-h_{x}\right)^{2}+h_{y}^{2}},
$$

where $w_{s}$ denotes the extrusion speed above any vents in the plane, $S=(L / H) \tan \phi$ is a measure of the slope relative to the fluid's typical aspect ratio (assumed to be order one, so that the slope must be sufficiently gentle), and

$$
B=\frac{\tau_{p}}{\epsilon \rho g H \cos \phi}
$$

is the 'Bingham number', a dimensionless measure of the yield stress ( $\rho$ is density and $g$ is gravity). Additionally $(\mathscr{U}, \mathscr{V})$ is the depth-integrated velocity field, and $s$ is the slope of the fluid surface.

We solve the equations on the domain $x_{u} \leqslant x \leqslant x_{d}$ and $y_{l} \leqslant y \leqslant y_{r}$. Unless otherwise stated, the boundary condition is that the gradient of $h(x, y, t)$ normal to the edge must vanish. The limits, $x_{l}, x_{u}, y_{l}$ and $y_{r}$ are chosen sufficiently large that the boundaries do not impede the flow. The initial conditions are designed for the particular kind of flow problem (that is, the scenarios (i)-(iii) described above), and will be given below. If the fluid occupies a compact area, the global integral of the evolution equation provides the mass conservation law,

$$
\frac{\mathrm{d}}{\mathrm{d} t} \int_{x_{u}}^{x_{d}} \int_{y_{l}}^{y_{r}} h(x, y, t) \mathrm{d} x \mathrm{~d} y=\int_{x_{u}}^{x_{d}} \int_{y_{l}}^{y_{r}} w_{s}(x, y, t) \mathrm{d} x \mathrm{~d} y \equiv Q,
$$

where $Q$ is the total mass flux of any sources (assumed constant in this study).

The yield criterion appears within the evolution equation through the function $Y$. There is a subtlety hidden in these equations associated with this function (Balmforth \& Craster 1999): because the surface is assumed to be stress-free, provided the fluid is flowing over the plane, there is a surface $z=Y(x, y, t)$ above which $\tau$ must fall below $\tau_{p}$. For $z>Y$, the theory appears to predict that the fluid is unyielded $-\mathrm{a}$ rigid plug flow - and so $z=Y$ is a yield surface. This prediction is incompatible with the fact that the fluid is spreading over the plane (that is, that there is divergence or convergence of the flow). Hidden at higher order is the resolution of this contradiction: above $z=Y$, the stresses are not actually below the yield value, but order $\epsilon$ above it. In other words, the level $z=Y(x, y, t)$ is really a 'fake' yield surface, and the upper region is a 'pseudo-plug' (to use the terminology of Walton \& Bittleston 1991).

Most previous expositions have considered one-dimensional versions of the model (2.4), obtained by considering either axisymmetrical extrusions on flat plates (Balmforth et al. 2000), or sheet flows without dependence on $y$ (Liu \& Mei 1989). The Newtonian limit of the theory $(n=1$ and $B=0)$ was considered by Huppert (1982) 
and Lister (1992). Steady solutions, with the further approximation that $S-h_{x} \approx S$, were given by Coussot \& Proust (1996).

Except in some special limits, we cannot solve the reduced model in closed form and we fall back on numerical computations. We use an adaptive scheme (Blom, Trompert \& Verwer 1996), designed to deal with sharp fronts; the accuracy of the code is verified by comparisons amongst runs with different resolutions and with one-dimensional solutions for expanding axisymmetrical domes (Balmforth et al. 2000). Two other points deserve mention. First, in the computations, it is necessary to smooth out the function $Y$; we adopt a regularization in the spirit of Appendix A of Balmforth et al. (2000). This particular regularization has little apparent effect. Second, in order to make the code run efficiently, it was necessary to add a small linear diffusion term, $\kappa\left(h_{x x}+h_{y y}\right)$, to the right of the evolution equation and 'pre-wet' the plane - that is, we choose initial fluid distributions of the form, $h(x, y, 0)=h_{I}(x, y)+h_{s}$, where $h_{I}(x, y)$ denotes any substantial localized initial volume, and $h_{s}=10^{-3}$ or smaller. The linear diffusivity $\kappa$ was selected to be as small as possible to minimize any associated diffusion whilst maximizing the operating efficiency of the code (typical values were $10^{-4}$ ). Based on grid refinement and variation of $\kappa$, we believe that the dynamics is largely unaffected by the linear diffusion, although the steep edges of the computed domes (the sharp contact between the dome and the pre-wetted region) are discernibly smoothed. One unappealing aspect of the computations is that the scheme is unable to access reliably the large-time solutions in which the yield surface becomes very low. However, this limit can be explored with the analytical theory described in $\S 4$.

Thus formulated, the model has the parameters $B$ and $S$, together with any others that describe the source $w_{s}$, and the initial or inflow profiles (see below). However, the characteristic lengthscales $H$ and $L$ are not prescribed at this stage, and so we have the freedom to select these scales to remove two of the parameters of the problem. We choose $S=1$ (unless the plane is horizontal, in which case an alternative scaling is needed) and fix either the incoming flux of material or the initial thickness. This leaves the Bingham number $B$ and power-law exponent $n$ as free parameters, together with any others determining the shape of any sources or initial profiles. However, we refrain from everywhere setting $S=1$ formally so that we have the option of considering horizontal planes. Most prior studies concentrate upon the Bingham model with $n=1$; for the purposes of illustration we use this value in all computations.

\section{Numerical solutions}

\subsection{Extrusions}

To follow lava-like extrusions from vents onto inclined planes, we take $h_{I}(x, y)=0$ and specify the source function,

$$
w_{s}=w_{s}(r)=\frac{1}{r_{*}^{2}}\left(1-\frac{r^{2}}{r_{*}^{2}}\right)^{2} \theta\left(r_{*}-r\right),
$$

where $r$ is the radial coordinate (with the origin located at the centre of the vent), $\theta(r)$ is the Heaviside step function, and $r *$ represents the vent radius. (This form ensures that the source flux is constant in time and independent of $r_{*}$.) We shall be mainly interested in flows for which the fluid extent is far larger than the vent, $r_{*} \ll 1$, which means that $B$ (and $n$ if it were used) are the most significant parameters for our extrusions, in line with the experiments performed by Osmond \& Griffiths (2001). 

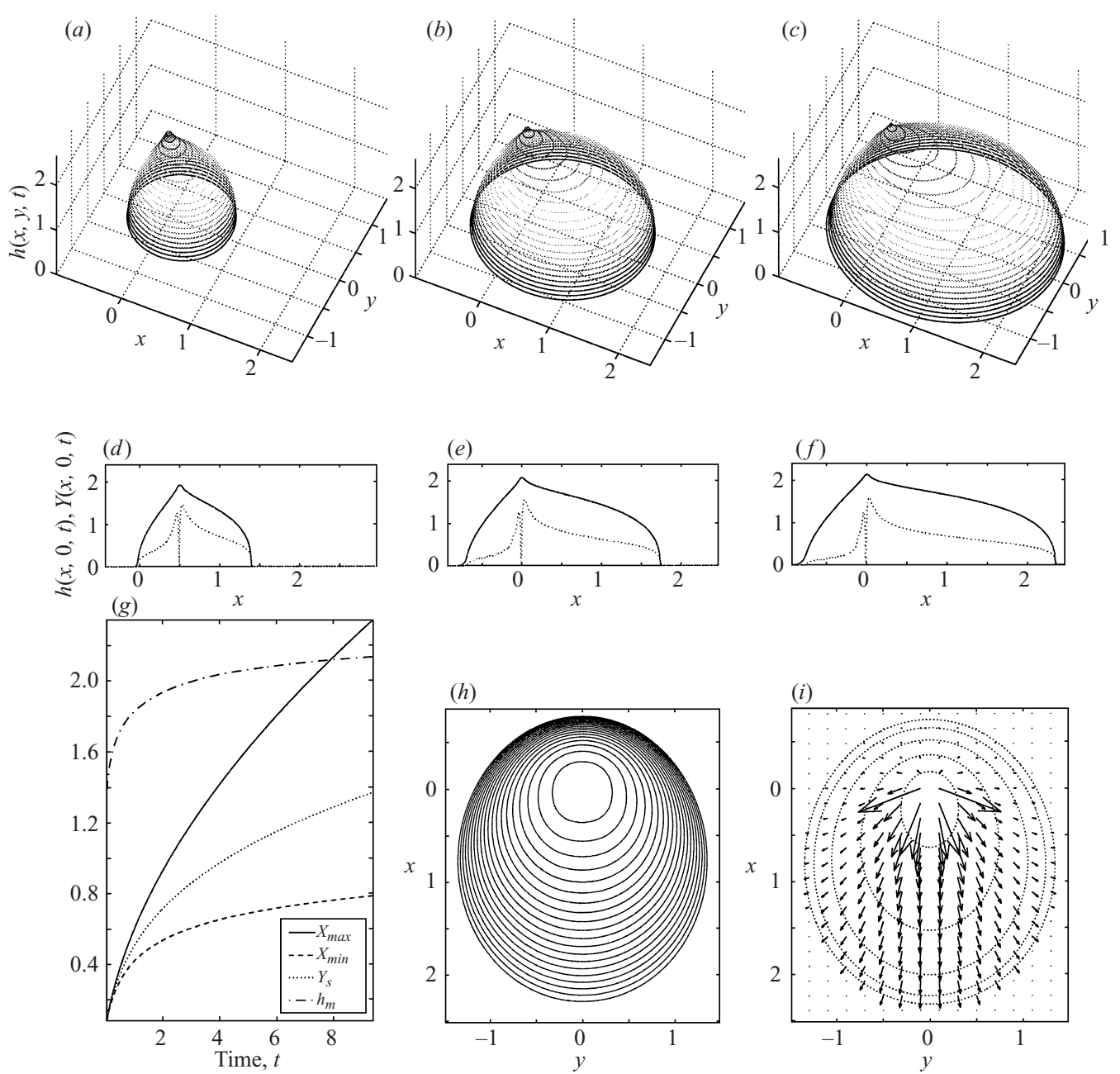

Figure 2. An extruded, slumped dome on an inclined plane, computed numerically using the thin-layer model with $B=1.33$. $(a-c)$ Three snapshots of the domes at times $1.88,5.64$ and 9.4, respectively. Directly below, $(d-f)$, are the corresponding height profiles (solid lines) and yield surfaces (dotted lines) along the midsection $(y=0)$. $(g)$ The evolution of the cross-stream half-thickness $\left(Y_{s}\right)$, the downslope and upslope lengths $\left(X_{\max }\right.$ and $\left.X_{\min }\right)$, and the maximum height $\left(h_{m}\right)$. (h) A sequence of curves showing the dome's edge (the curves show the edge every 0.38 time units), and (i) vectors indicating the depth-averaged velocity field, $h^{-1}(\mathscr{U}, \mathscr{V})$, at $t=9.4$ (the dotted curves are contours of constant height).

\subsubsection{Dome shapes}

We illustrate the evolution of an extrusion onto a sloping plane in figure 2 . The dome has $B=1.33$ and $n=1$ (with $r_{*}=0.05$ for the vent radius); as mentioned above, we always take $S=1$ unless the plane is flat. Three snapshots of the dome are shown. The midsection profiles of the dome, together with the corresponding yield surface, $Y(x, y=0, t)$, illustrate how this dome has a pseudo-plug and a yielding region of roughly equal volume. Also displayed are some characteristic measures of the dome's size and shape: the maximal height, $h_{m}(t)$, the half-width, $Y_{s}(t)$, and the maximum lengths upstream and downstream of the vent, $X_{\min }(t)$ and $X_{\max }(t)$, 

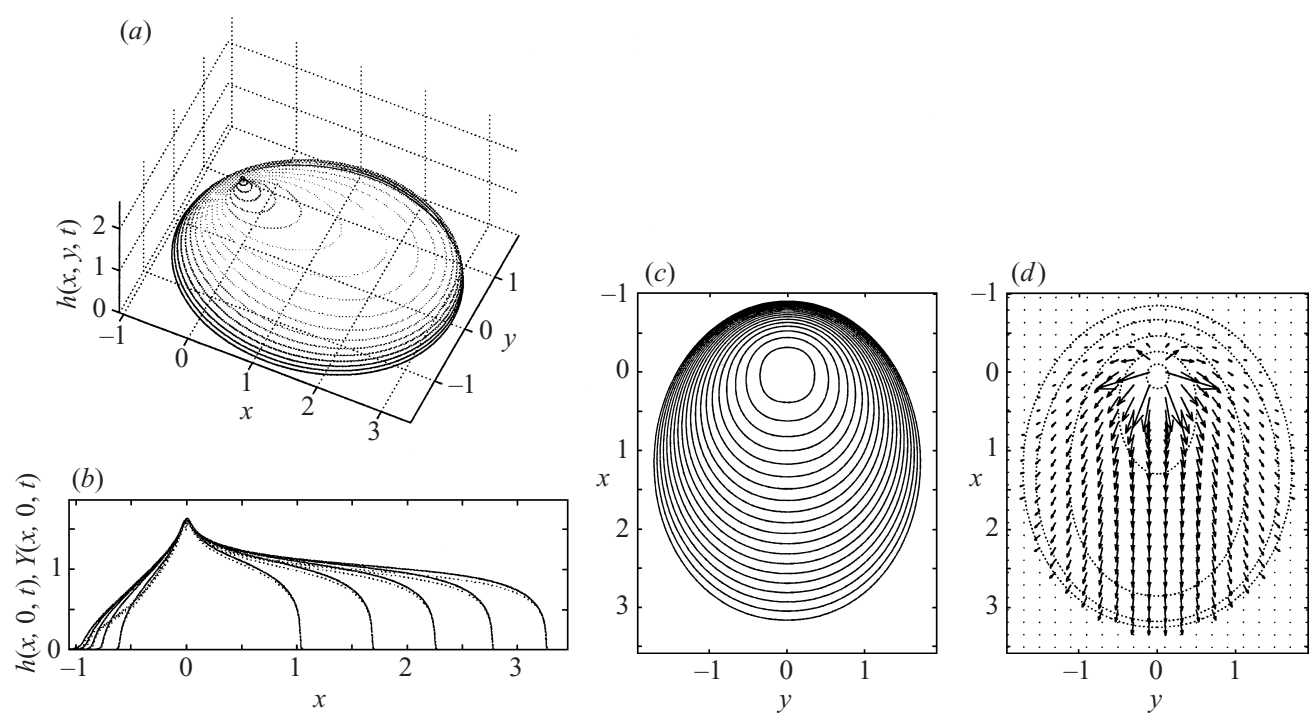

Figure 3. A dome with $B=0.067$. (a) A snapshot of the dome at $t=9.4$. ( $b$ ) Five snapshots of the midsection profile (solid lines) and corresponding yield surface (dotted lines) at times 1.88, 3.76, 5.64, 7.52 and 9.4. (c) A sequence of curves showing the dome's edge (the curves show the edge every 0.38 time units), and $(d)$ vectors of the depth-averaged velocity field at the final time (with superposed contours of constant height).

respectively. The lengths all diverge from one another after a short time, revealing the influence of the background slope. The effect of the downslope slumping is illustrated further by a succession of images of the dome edge, and by the vectors of the depth-averaged velocity field, $h^{-1}(\mathscr{U}, \mathscr{V})$, at the final time.

For comparison, we show domes with $B=0.067$ and $B=26.6$ in figures 3 and 4 . The first dome is almost Newtonian, whereas the second is dominated by its yield stress (as illustrated by the pseudo-plug which occupies almost the whole dome and $Y \ll h$ ). After an initial period, the nearly Newtonian dome spreads mainly downhill and the upper section of the dome is close to stationary with an almost flat surface ( $h_{x} \approx S=1$ ). The dome with larger- $B$ is most symmetric, an obvious consequence of the greater yield strength, and compares well with the results predicted by the large- $B$ theory described in $\S 4$.

\subsubsection{Similarity solutions and dome scalings}

For point sources, we can look for similarity solutions that directly uncover how the global dome characteristics $\left(h_{m}, X_{\max }, X_{\min }\right.$ and $\left.Y_{s}\right)$ vary with time. Let

$$
x=t^{\gamma} \xi, \quad y=t^{\delta} \eta, \quad h(x, t)=t^{1-\gamma-\delta} \varphi(\xi, \eta),
$$

which ensures that the mass conservation law becomes

$$
Q=\int_{-\infty}^{\infty} \int_{-\infty}^{\infty} \varphi(\xi, \eta) \mathrm{d} \xi \mathrm{d} \eta
$$

(for constant mass influx). The evolution equation can then be written in the form

$$
\varphi-\gamma(\xi \varphi)_{\xi}-\delta(\eta \varphi)_{\eta}+v_{\xi}+v_{\eta}=0,
$$


(a)
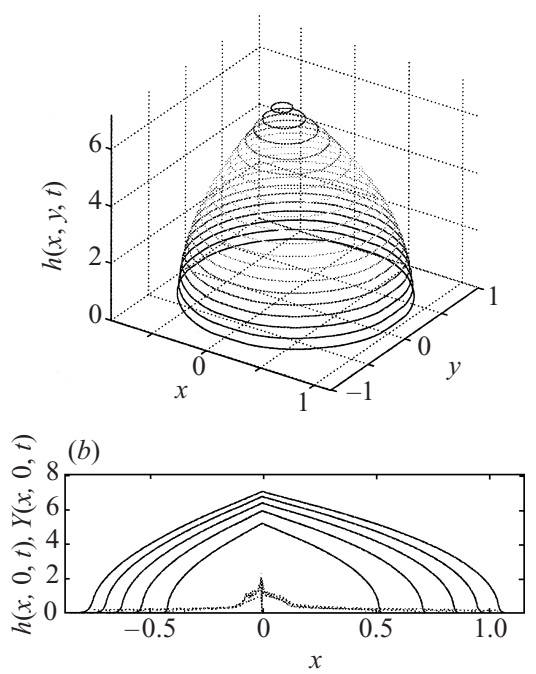
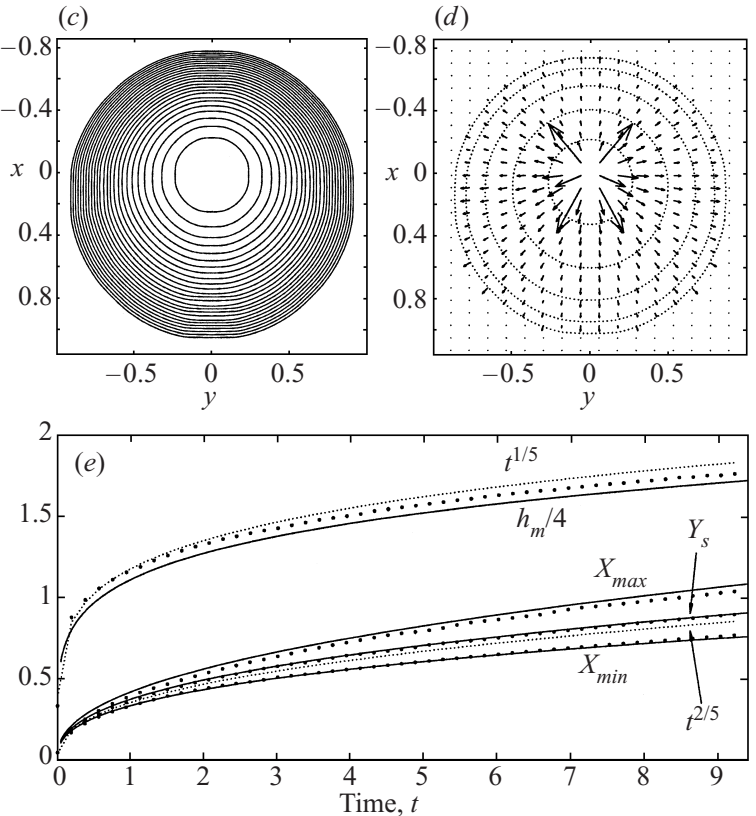

Figure 4. A similar picture to figure 3 , but for a dome with $B=26.6$. The additional picture $(e)$ shows the height (divided by four, for clarity) and the characteristic lengths. The points show the numerical computations and the solid lines indicate the analogous results for the large- $B$ theory of $\S 4$. The dotted lines show the characteristic scalings, $t^{1 / 5}$ and $t^{2 / 5}$.

where

$$
\begin{aligned}
\left(\begin{array}{l}
v \\
v
\end{array}\right) & =-\frac{n \Upsilon^{1+1 / n} \varsigma^{1 / n-1}[\varphi(2 n+1)-n \Upsilon]}{(n+1)(2 n+1)}\left(\begin{array}{c}
\varphi_{\xi}-t^{2 \gamma+\delta-1} S \\
t^{2 \gamma-2 \delta} \varphi_{\eta}
\end{array}\right) t^{\alpha}, \\
\Upsilon & =\varphi-\frac{B}{\varsigma} t^{3 \gamma+2 \delta-2}, \quad \varsigma=\sqrt{\left(\varphi_{\xi}-t^{2 \gamma+\delta-1} S\right)^{2}+t^{2 \gamma-2 \delta} \varphi_{\eta}^{2}}
\end{aligned}
$$

and $\alpha=[2(n+1)-(n+2) \delta-(2 n+3) \gamma] / n$.

The extrusion of the dome begins at $t=0$. We look for a nearby small-time similarity solution by balancing the time derivative (which generates the first three terms on the left of (3.4)) with the largest terms from the fluxes, $v_{\xi}+v_{\eta}$, when $t^{-1} \gg 1$. This fixes $\alpha=0$ (or $\gamma=2(n+1) /(3 n+5))$ and $\delta=\gamma$. Furthermore,

$$
\left(\begin{array}{l}
v \\
v
\end{array}\right) \rightarrow-\frac{n \varphi^{2+1 / n} \varsigma^{1 / n-1}}{(2 n+1)}\left(\begin{array}{c}
\varphi_{\xi} \\
\varphi_{\eta}
\end{array}\right) \quad \text { and } \varsigma \rightarrow \sqrt{\varphi_{\xi}^{2}+\varphi_{\eta}^{2}}
$$

The equations can then be solved on transforming to polar coordinates, whereupon one recognizes the solution as that for an axisymmetrical power-law fluid on a horizontal plane (Balmforth et al. 2000).

As time increases, the terms omitted in arriving at (3.7) grow and eventually cannot be neglected, indicating that there must be a transition in the dome scalings. The slope-dependent terms of (3.4)-(3.6) become important for $t$ order one. If these terms are the first to appear, then we expect a transition to a slope-dominated state (as in Lister 1992). However, the term stemming from the yield stress in $\Upsilon$ can also become important for times $t \sim B^{1 /(2-3 \gamma-2 \delta)} \equiv B^{-(5+3 n) / 4 n}$. If these terms are the first to appear, we expect a transition to a yield-stress-dominated state. Hence, if $B \ll 1$, there is a slope-induced transition, whereas for $B \gg 1$, the yield stress first becomes 


\begin{tabular}{lcccc}
\hline & Downslope & Upslope & Half-width, & Maximal \\
length, $X_{\max }$ & length, $X_{\min }$ & $Y_{s}$ & \\
& & Similarity solution, $, \quad \ll 1, n \neq 1$ & \\
Early & $2(n+1) /(3 n+5)$ & $2(n+1) /(3 n+5)$ & $2(n+1) /(3 n+5)$ & $(1-n) /(3 n+5)$ \\
Late & $(5 n+2) /[3(2 n+1)]$ & $-n /[3(2 n+1)]$ & $1 / 3$ & $-n /[3(2 n+1)]$ \\
& & & \\
Early & $1 / 2$ & Similarity solution, $B \ll 1, n=1$ & 0 \\
Late & $7 / 9$ & $1 / 2$ & $1 / 2$ & $-1 / 9$ \\
& & $-1 / 9$ & $1 / 3$ & $1 / 5$ \\
Early & $2 / 5$ & Yield-stress-dominated dome & 0 \\
Late & 1 & $2 / 5$ & $2 / 5$ & 0
\end{tabular}

TABLE 1. The temporal power-law scalings expected from the similarity solutions for the downslope length, $X_{\max }$, the upslope length, $X_{\min }$, the half-width, $Y_{s}$, and the maximal height, $h_{m}$. Also included are the scalings expected from the theory of $\S 4.1$.

important. Yield-stress-dominated domes are considered in $\S 4.1$, so we entertain the first possibility here - a transition due to slope.

We rewrite (3.5)-(3.6):

$$
\begin{gathered}
\left(\begin{array}{l}
v \\
v
\end{array}\right)=\frac{n \Upsilon^{1+1 / n} \tilde{\varsigma}^{1 / n-1}[\varphi(2 n+1)-n \Upsilon]}{(n+1)(2 n+1)}\left(\begin{array}{c}
S-t^{1-2 \gamma-\delta} \varphi_{\xi} \\
-t^{1-3 \delta} \varphi_{\eta}
\end{array}\right) t^{\beta}, \\
\Upsilon=\varphi-\frac{B}{\tilde{\varsigma}} t^{\gamma+\delta-1}, \quad \tilde{\varsigma}=\sqrt{\left(S-t^{1-2 \gamma-\delta} \varphi_{\xi}\right)^{2}+t^{2-2 \gamma-4 \delta} \varphi_{\eta}^{2}}
\end{gathered}
$$

and $\beta=[(2 n+1)(1-\gamma)-(n+1) \delta] / n$. Now we look for a solution with $\beta=0, \delta=1 / 3$ and $\gamma=(5 n+2) / 3(2 n+1)$. In this case,

$$
\left(\begin{array}{l}
v \\
v
\end{array}\right) \rightarrow \frac{n \Upsilon^{1+1 / n} S^{1 / n-1}[\varphi(2 n+1)-n \Upsilon]}{(n+1)(2 n+1)}\left(\begin{array}{c}
S \\
-\varphi_{\eta}
\end{array}\right) \quad \text { and } \quad \tilde{\varsigma} \rightarrow S
$$

Provided that the yield-stress term remains negligible, we must then solve the equation,

$$
\varphi-\gamma(\xi \varphi)_{\xi}-\frac{1}{3}(\eta \varphi)_{\eta}+\frac{n S^{1 / n}}{2 n+1}\left(\varphi^{2+1 / n}\right)_{\xi}-\frac{n S^{1 / n-1}}{2 n+1}\left(\varphi^{2+1 / n} \varphi_{\eta}\right)_{\eta}=0,
$$

to specify fully the solution. Note that the scalings predict a spreading flow whose thickness declines with time $\left(h \sim t^{-n / 3(2 n+1)} \varphi\right)$. This solution can only describe the downslope portion of the slumping dome. Upslope of the vent, the axisymmetrical expansion subsides leaving an almost stagnant pool of fluid with a flat surface (Lister 1992). Thus the upper edge of the dome comes to rest (cf. figure 3), and then recedes to keep the surface flat once the thickness begins its decline.

Equation (3.11) is still not the end of the story because, even when the slope dominates, the yield-stress term is not subdominant: $B t^{\delta+\gamma-1} \rightarrow B t^{n / 3(2 n+1)}$. In other words, yield stresses always grow to induce another transition to a yield-stressdominated state. That transition occurs for $t \sim B^{-3(2 n+1) / n}$. We summarize the results in table 1, which lists the scalings that are predicted for the maximal height, $h_{m}$, and the characteristic dome lengths, $X_{\min }, X_{\max }$ and $Y_{s}$. The results for yield-stress-dominated domes are derived in $\S 4.1$. 


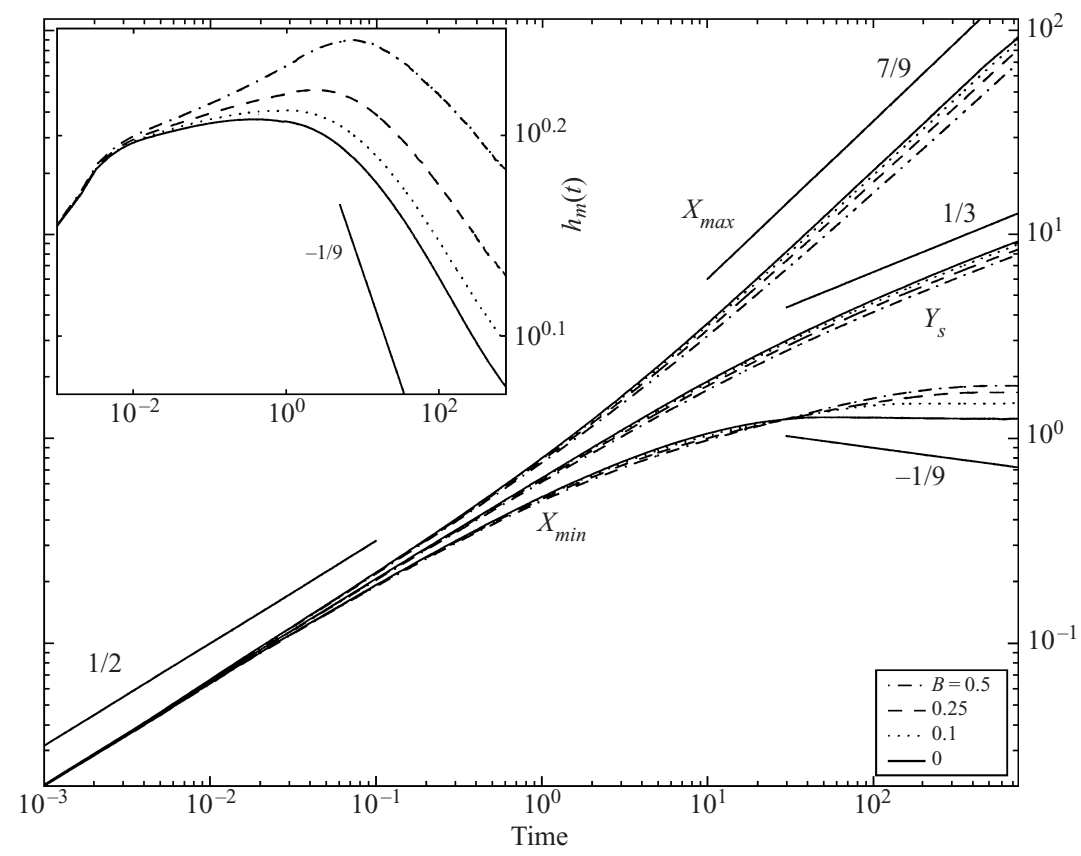

FIGURE 5. Evolution of the dome's characteristic lengths and height for different (but small) values of $B$ and $n=1$. The domain in these computations expands with time. The increase of the domain is determined by the advance of the dome's edges (that is, by approximating $X_{\min }, X_{\max }$ and $Y_{s}$ by linear functions of time, and using the corresponding speeds to advance the upper, lower and lateral boundaries of the domain). The vent is fixed in the computational space, and so corresponds to an expanding vent in physical space; the expansion of the vent keeps the ratio of the source radius to dome edge roughly constant, and avoids any gradual loss of resolution at long times. For $t<10^{-2}$ the linear rise of $h_{m}(t)$ reflects the initial emplacement of the fluid.

We compare the scaling predictions for smaller $B$ with numerical results in figure 5 . This illustrates how the axisymmetrical short-time scalings $\left(X_{\min } \sim X_{\max } \sim Y_{s} \sim t^{1 / 2}\right)$ change due to the effect of the background slope for times of order unity. Thereafter, the more Newtonian domes pass to the long-time scaling predicted above, in which $X_{\max } \sim t^{7 / 9}$ and $Y_{s} \sim t^{1 / 3}$. A slight disagreement between the computations and the similarity analysis is that the upslope length, $X_{\min }$, slows to rest and then begins to subside back down the slope for $B=0$, but never reaches the $t^{-1 / 9}$ scaling. Also, the maximal height varies differently with time. The domes with larger $B$ show increasing deviations from the Newtonian scalings, mainly for times larger than unity, illustrating the emergence of yield stresses. Particularly noticeable is the lack of subsidence in the upslope length $X_{\min }$ - the upper regions of the fluid become stationary and held at the yield stress, rather than slowly slump downhill.

\subsection{Rivulets}

To model flows fed by an upstream reservoir, we take $w_{s}=0$ and modify the boundary condition along the upper edge of the plane, $x=x_{u}=0$. Here, we assume that the reservoir feeds the flow through an aperture of a given shape. We take the smooth function $h(0, y, t)=h_{u}(y)=\exp \left[-\left(y / y_{*}\right)^{2}\right]$, where $y_{*}$ is the characteristic half-width. The Gaussian shape is convenient, but not significant - one could use a function more closely resembling an experimental set-up, if desired. We start the computation with the initial condition $h_{I}(x, y)=h_{u}(r)\left(r^{2}=x^{2}+y^{2}\right)$. 

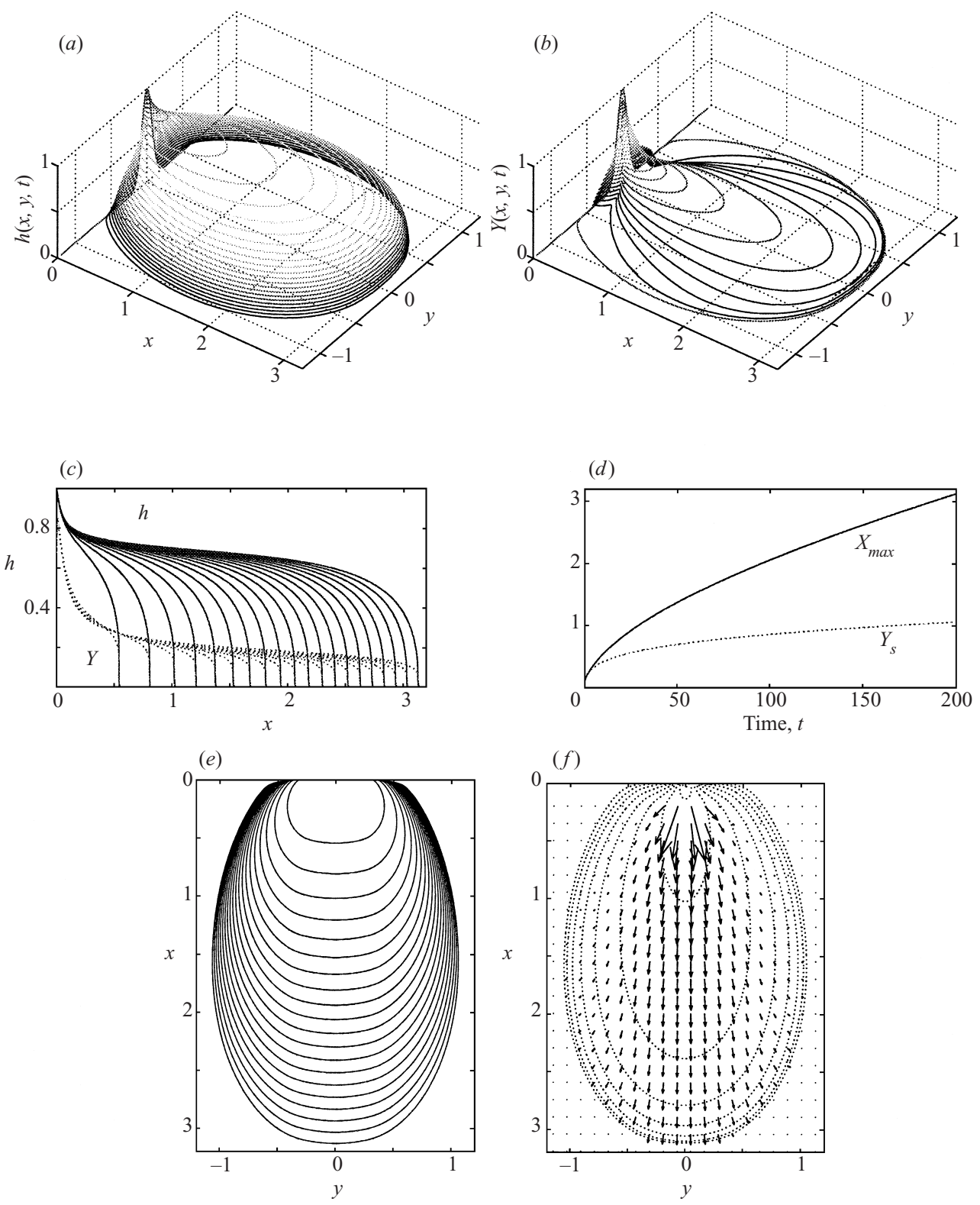

FIGURE 6. Rivulet forming from an outflow from an aperture with $B=1 / 2$ and $y_{*}=0.1$. (a) The height contours and $(b)$ yield surface at $t=200$ (the outermost contour in $(b)$ shows the fluid edge). Also shown are $(c)$ the height profiles (solid and dashed lines) and yield surfaces (dotted lines) along the midsection $(y=0)$ for times $t=0,10,20, \ldots, 200$, and $(d)$ the time evolution of the width $Y_{s}$ (dotted line) and downstream length $X_{\max }$ (solid line). (e) A sequence of images of the edge of the fluid taken every 10 time units, and $(f)$ vectors of the depth-averaged velocity field at $t=200$ (with superposed height contours).

A developing rivulet is shown in figure 6. The fluid initially flows out both down and across the plane, creating a structure that is much wider than the aperture. The cross-slope flow subsequently slows and a tongue of downhill moving fluid is created. The lateral portions of the flow near the aperture become almost stationary (as indicated by the relatively low yield surface and the vectors of the depth-integrated 


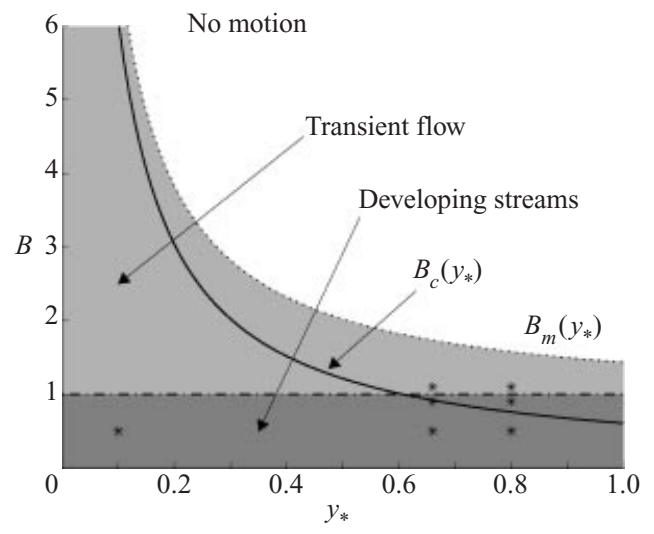

FIGURE 7. Regime diagram on the $\left(y_{*}, B\right)$-plane for rivulets with the initial and $y=0$ boundary condition, $h(x, y, 0)=\exp \left[-\left(x^{2}+y^{2}\right) / y_{*}^{2}\right] . B_{m}\left(y_{*}\right)$ is the maximum value of $s h$ at $t=0$ (the greatest initial gravitational stress); if $B>B_{m}\left(y_{*}\right)$, the initial profile is held in position by the yield stress. The horizontal line, $B=S h_{\max } \equiv 1$, marks the limit below which rivulets expand inexorably into flowing streams. The two lines, $B=B_{m}\left(y_{*}\right)$ and $B=1$, divide the parameter plane into three characteristic regimes (no motion, transient flows, developing streams). The curve $B_{c}\left(y_{*}\right)$ is the boundary below which the characteristics solution of $\S 4.2$ is not valid. The stars locate the parameter settings used in the computations.

velocity field at the end of the computation), forming what one might refer to as 'levees'. However, the levees are not completely stationary and the flow does not evolve to constant width (as assumed by Hulme 1974), but continues to widen with time and distance downstream. This is what one expects from a consideration of the possible steady flow profiles (Coussot \& Proust 1996). The overall shape of the flow is similar to the experimental results presented by Coussot \& Proust (1996).

An important feature of the rivulets formed in this way is that they are not constant-mass-flux extrusions. In fact, because the thickness is prescribed on the boundary but the surface gradient is free to evolve, the mass flux varies with time. Indeed, if $B$ is sufficiently large, the initial state can be supported by the yield stress without moving at all (so the mass flux remains zero). Conversely, when $B$ is small, the yield stress is never large enough to support the fluid at the aperture itself, and so material always flows into the domain to seed a stream. Between these two limits, the fluid at the aperture can be below the yield stress, and initially stationary, but downslope the yield stress exceeded. The fluid then flows initially, but slumps to rest when surface gradients decline and the associated stresses fall beneath the yield value. In this circumstance, there can be a transient mass flux.

To predict the evolution of a particular configuration, we first estimate the dimensionless maximal stress exerted by gravity on the initial configuration (equivalent to the maximum value of $s h$ over the domain), denoted $B_{m}\left(y_{*}\right)$. If $B>B_{m}\left(y_{*}\right)$, the yield stress is not exceeded, and the fluid is held in position. On the other hand, the gravitational stress is smallest, and equals unity, at $x=y=0$. Thence, the fluid must flow at the aperture if $B<1$. We therefore predict stationary states for $B>B_{m}\left(y_{*}\right)$, and flows that inexorably expand into streams for $B<1$. The intermediate cases, with $1<B<B_{m}\left(y_{*}\right)$, can flow initially, but brake to a halt. Figure 7 sketches out the three regimes.

The example shown in figure 6 corresponds to a flow that continues to expand into a stream. A second example, with $B=1.1$ and $y_{*}=0.66$, is shown in figure 8 . This 
flow slumps initially but subsequently decelerates to rest (illustrating the intermediate case). The evolving structure in this picture contains portions over which the yield stress is never exceeded, and therefore never flow. The remainder of the fluid slumps downslope and approaches a stationary state that can be computed by the methods of $\S 4.2$. The approach of the midsection to the expected final stationary profile is also illustrated in the figure; the expected profile is given implicitly by

$$
S x=h-1-\frac{B}{S} \log \left(\frac{B-S}{B-S h}\right) .
$$

A collection of computations (corresponding to the stars in figure 7) showing how downstream length varies with time is displayed in figure 9; this picture illustrates the deceleration of runs with $B>1.1$ (intermediate cases), and the continued flow of those with $B<1$ (forming streams).

\subsection{Slumps}

To simulate the release of a finite volume of fluid, we take $w_{s}=0$ and prepare the fluid with the initial profile $h_{I}(x, y)=\exp \left[-\left(x / x_{*}\right)^{6}-\left(y / y_{*}\right)^{6}\right]$; this is a convenient illustrative profile, being both smooth and having adjustable parameters, $x_{*}$ and $y_{*}$, through which we can vary the volume and shape. Slumps with $B=0.5$ and $n=1$ are illustrated in figure 10 ; the two cases have initial profiles with $\left(x_{*}, y_{*}\right)=(3 / 4,1 / 4)$ and $\left(x_{*}, y_{*}\right)=(1 / 2,1 / 2)$. The domes slump primarily downslope, together with a slight uphill slump, and yield almost everywhere. Eventually, the fluid nearly comes to rest with a structure held almost exactly at the yield stress: $Y=h-B / s \approx 0$. The expected final stationary state can again be constructed using theory described in $\S 4.2$. Of course, the presence of linear diffusion in the numerical scheme means that the slumps never truly come to rest in the computations, but always are subject to a residual spreading. This is particularly evident near the edges of the slumped domes in the figure (residual spreading at the front is also obvious in figure 8 for a decelerating rivulet).

Even though the initial volumes of the two numerical experiments are roughly the same, the slumped domes are quite different. This is because the final state contains a ridge along which the surface gradient is almost discontinuous, and is not a dome with a single pinnacle. The ridges are held at the yield stress and have varying lengths depending on the initial profile. The ridge roughly divides the part of the fluid that slumped upslope from the part that slumped downslope. This feature implies a pronounced dependence on the initial profile which must be removed in order to measure the rheology in any slump test.

\section{Yield-stress-dominated domes and slumped deposits}

When $B \gg 1$, extruded domes become dominated by the yield stress. In this situation, the pseudo-plug occupies most of the dome and $Y \ll 1$ (see figure 4). The condition $Y=0$ also characterizes the final shape of fluids that slump to rest from an initial configuration (or at least the part that yielded during the motion), regardless of the value of $B$. These considerations can be turned into a common analytical theory for the corresponding shapes adopted by the fluid.

From equation (2.5), a vanishing yield surface implies

$$
\left(S-h_{x}\right)^{2}+h_{y}^{2}=B^{2} / h^{2},
$$

which determines $h$. For slumped deposits, the equality (4.1) is exact and finding 

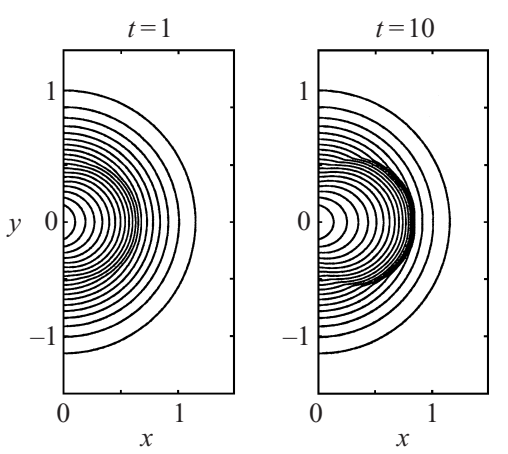

(a)

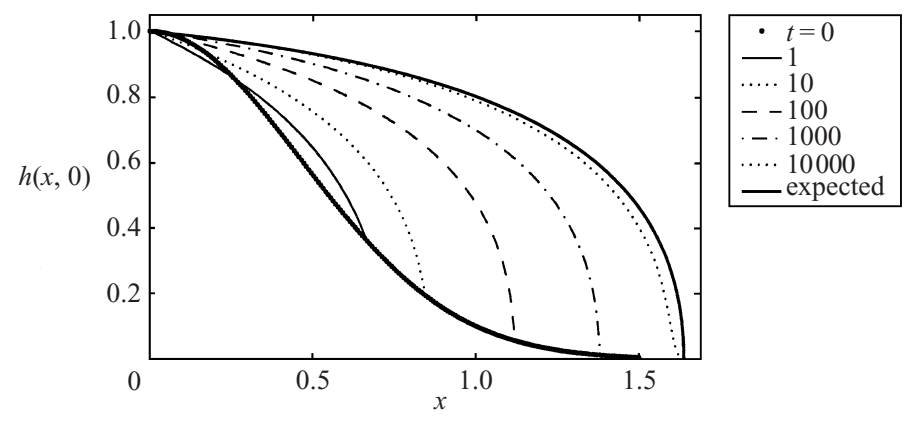

FiguRE 8. (a) Four snapshots of a rivulet spreading out from an aperture with $y_{*}=0.66 . B=1.1$. Contour levels begin at $h=0.1$ and then increase to unity in steps of 0.05 . (b) Five snapshots of the midsection. The initial condition is also shown, as is the expected final profile based on the theory of $\S 4.2$, given by $(3.12)$.

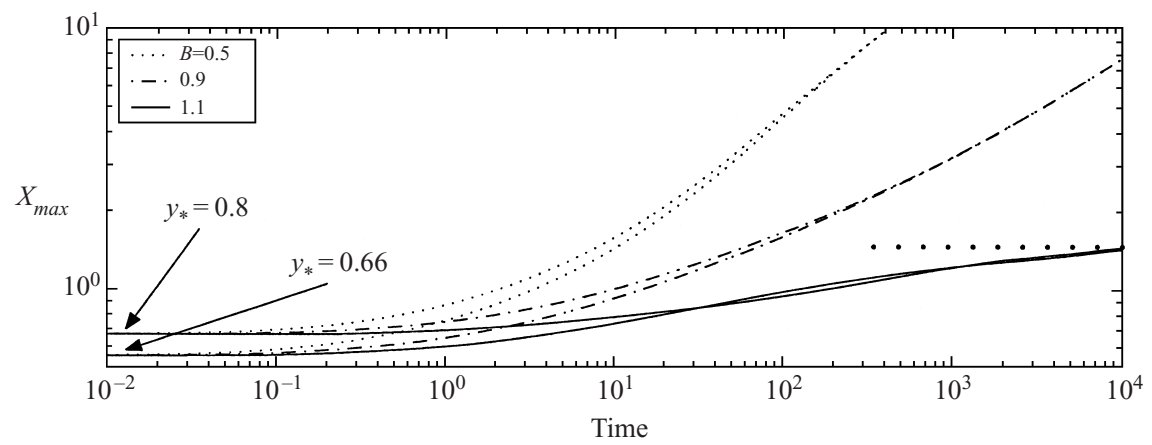

FiguRE 9. The progression of the downslope length, $X_{\max }$, with time for several rivulets with varying $B$ and $y_{*}$. The dots display the expected limiting value for $B=1.1$, which is given by $S X_{\max }=(B / S) \log [B /(B-S)]-1 \approx 1.64($ with $S=1)$.

its solution ends the story. (In fact, the theory for slumped, stationary structures is very general, applying to any yield-stress fluid whether or not it is described by the Herschel-Bulkley model.) But this equation is only an approximation for yield-stressdominated extrusions because a slender yielding region still exists adjacent to the plane which allows the domes to expand slowly. In this case, (4.1) is the leading-order 

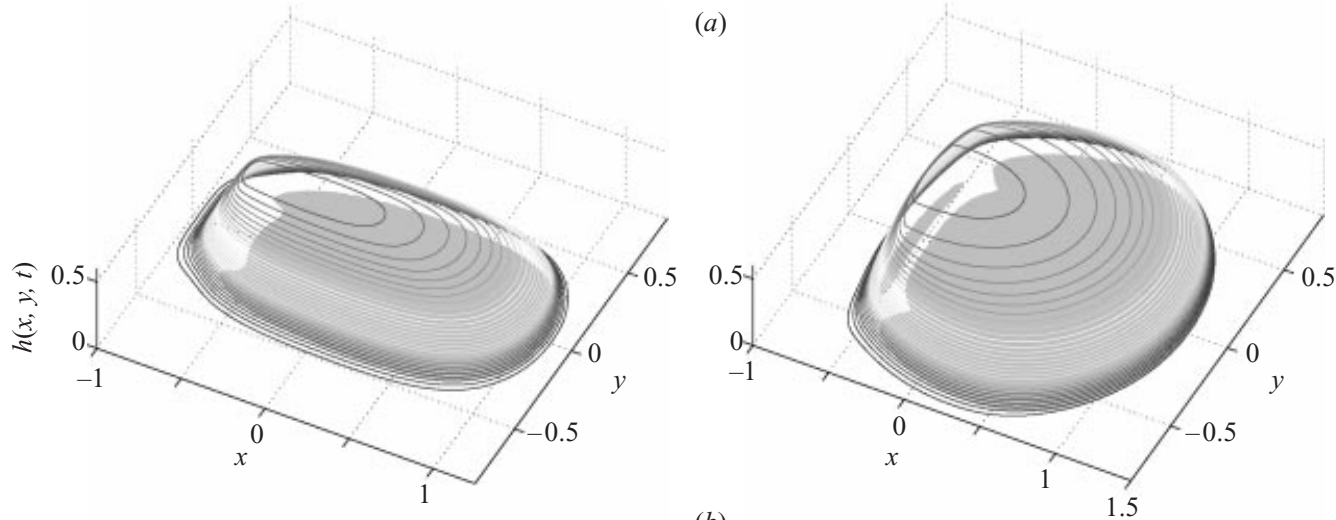

(b)
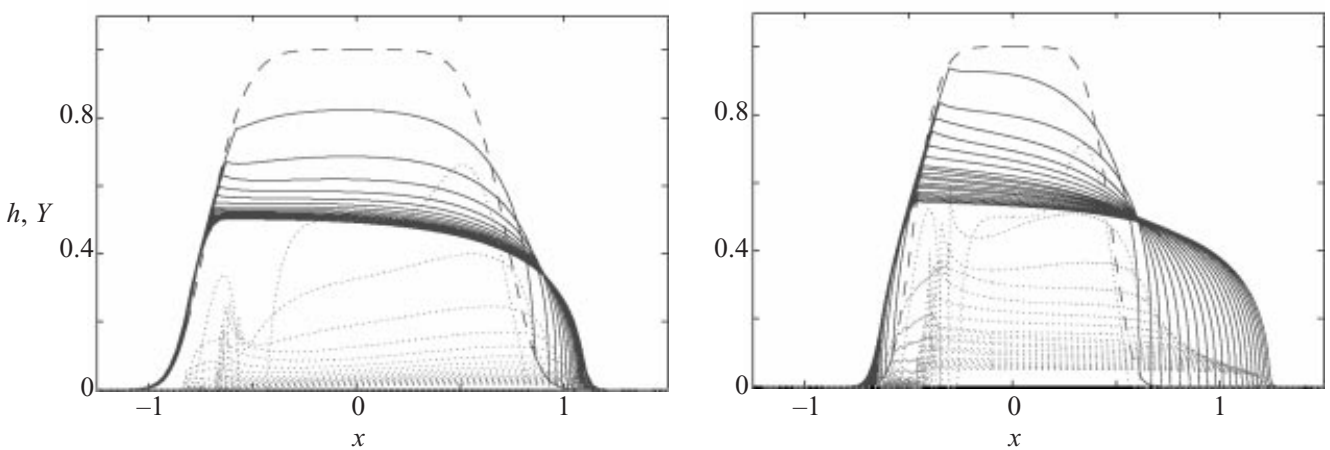

FiguRE 10. Domes created by the slump of a fixed volume. $B=1 / 2$. (a) The shape of two domes with different initial profiles $\left(\left(x_{*}, y_{*}\right)=(3 / 4,1 / 4)\right.$ and $\left.\left(x_{*}, y_{*}\right)=(1 / 2,1 / 2)\right)$ at $t=100$. The shaded region shows the area in which $Y>0$. (b) The corresponding height profiles (solid and dashed lines) and yield surfaces (dotted lines) along the midsection $(y=0)$ for times $t=j^{2} / 4$ with $j=0,1,2, \ldots, 20$.

equation in an asymptotic expansion for $B \gg 1 \uparrow$. The yield-stress-dominated domes evolve quasi-statically by marching through a succession of states described by (4.1). Later, in $\S 4.1$, we calculate in detail the dome evolution.

Equation (4.1) can be solved using the general technique of characteristics commonly called Charpit's method (Sneddon 1957). In this method, we solve the partial differential equation (4.1) by computing its characteristic curves. Each curve is parameterized by a variable $\tau$ and is constructed by setting $p=h_{x}$ and $q=h_{y}$, and then solving some ordinary differential equations for $x, y, h, p$ and $q$ that follow from the governing partial differential equation. Here, the characteristic equations can be written in the form,

$$
\dot{x}=2(S-p), \quad \dot{y}=-2 q, \quad \dot{h}=2 p(S-p)-2 q^{2}, \quad \frac{\dot{p}}{p}=\frac{\dot{q}}{q}=\frac{2 B^{2}}{h^{3}},
$$

where the dot denotes differentiation with respect to $\tau$. With the solution to the characteristic equations in hand, we may reconstruct the general solution for $h$ by eliminating $\tau$ and any constants of integration.

$\dagger$ The analysis can be recast in the form of a conventional asymptotic theory using the rescalings, $x=B \tilde{x}, y=B \tilde{y}, h=B \tilde{h}, Y=B^{-2 n /(n+1)} \tilde{Y}$ and $t=B^{3} \tilde{t}$. Because we only need the leading order, we omit these rescalings here. Note, however, that $B / h$ remains order one in the limit. 
Two relations follow immediately from (4.2):

$$
p=a q, \quad x-a y=2 S \tau,
$$

where the constant of integration, $a$, is a property of each characteristic curve, and we have set $x=y=0$ at $\tau=0$. Hence, $\tau=(x-a y) / 2 S$. We next relate $h$ to $p$ using (4.1) and then integrate $\mathrm{d} x / \mathrm{d} h=\dot{x} / \dot{h}$ and $\mathrm{d} y / \mathrm{d} h=\dot{y} / \dot{h}$, to find

$$
S\left(y-y_{0}\right)=\sqrt{b^{2}+a^{2} b^{2}-h^{2}}-b \sqrt{1+a^{2}}
$$

and

$$
S\left(x-x_{0}\right)=h-\frac{b}{2} \log \left[\frac{\Delta(h)}{\Delta(0)}\right]
$$

where $b=B / S$,

$$
\Delta(h)=\left(\frac{a b+\sqrt{b^{2}+a^{2} b^{2}-h^{2}}}{a b-\sqrt{b^{2}+a^{2} b^{2}-h^{2}}}\right)\left(\frac{b+h}{b-h}\right)
$$

and $\left(x_{0}, y_{0}\right)$ denotes the curve parameterizing the fluid edge. These relations provide the solution in $y \geqslant 0$. Identical formulae, but with an additional minus sign on the left of (4.4), provide the solution in $y<0$, although the curve $\left(x_{0}, y_{0}\right)$ is different if the structure is not symmetrical about $y=0$.

At this stage, we can build the complete solution given the locus $\left(x_{0}, y_{0}\right)$ of the edge of the fluid. Alternatively, we may try to specify the solution by fixing $h$ along a given curve that intersects all the characteristics. For domes extruded from a point source, specifying the apex of the dome provides the most concise form of the solution. This is the line we take below in order to construct the history of a yield-stress-dominated extrusion. To compute the final stationary states of rivulets and slumps, however, we fix $h$ along a curve, as described further in $\S 4.2$.

\subsection{Extruded domes}

On taking the apex of the dome to lie at the origin and have height $h=h_{m}$, we find

$$
S y_{0}=b \sqrt{1+a^{2}}-\sqrt{b^{2}+a^{2} b^{2}-h_{m}^{2}}
$$

and

$$
S x_{0}=-h_{m}+\frac{b}{2} \log \left[\frac{\Delta\left(h_{m}\right)}{\Delta(0)}\right],
$$

which defines the edge, $\left(x_{0}, y_{0}\right)$, in terms of the dome height, $h_{m}$. At this point, $h_{m}$ is a parameter in the solution. For a truly stationary dome, we should take $h_{m}=1$. In a yield-stress-dominated extrusion, however, $h_{m}$ evolves and this is how time enters the large- $B$ problem.

Several features of the slumped dome can be explicitly identified. The median of the slumped dome $(y=0)$ is given by $a \rightarrow \pm \infty$, and provides the profile of the midsection,

$$
S x= \begin{cases}h-h_{m}+b \log \left[(b-h) /\left(b-h_{m}\right)\right], & a \rightarrow \infty \\ h-h_{m}+b \log \left[\left(b+h_{m}\right) /(b+h)\right], & a \rightarrow-\infty,\end{cases}
$$

as in Osmond \& Griffiths (2001) and Coussot et al. (1996) (this is also the same profile as a line source - see Appendix B and Liu \& Mei 1989). For $a=0$, on the other hand, we obtain

$$
S x=h-h_{m}-\frac{b}{2} \log \left[\frac{\left(b-h_{m}\right)(b+h)}{\left(b+h_{m}\right)(b-h)}\right], \quad S y=\sqrt{b^{2}-h^{2}}-\sqrt{b^{2}-h_{m}^{2}} .
$$




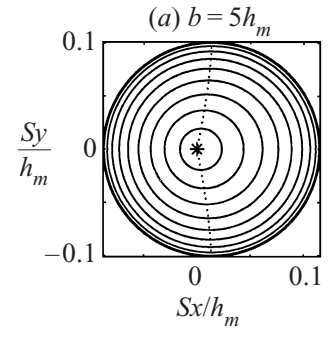

(d) $b=1.01 h_{m}$

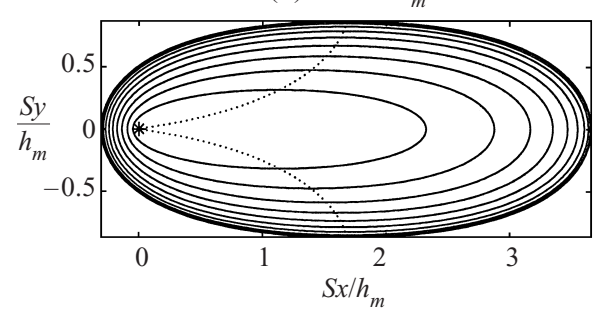

(b) $b=2 h$

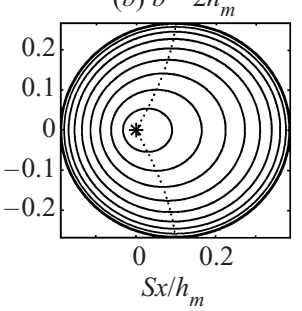

(c) $b=1.1 h$

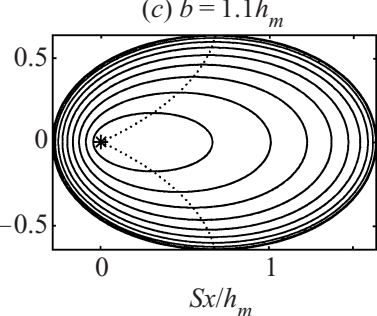

(e) $b \rightarrow h_{m}$

FiguRE 11. Contours of constant height (spaced by $h_{m} / 10$ ) for yield-stress-dominated domes on sloping planes. The dotted curves show the $a=0$ characteristic that connects to the widest part of the dome $(a-d)$. Contours of constant height for the limiting shape as $b \rightarrow h_{m}(e)$.

This second curve has furthest lateral extension and allows us to identify the width of the dome: $S Y_{s}=b-\sqrt{b^{2}-h_{m}^{2}}$. In the limit $S \rightarrow 0$, the characteristics become straight radial spokes, and by setting $a=\cot \theta$, where $\theta$ is the polar angle, one deduces $x=a y$ and the familiar axisymmetrical result, $h=\sqrt{h_{m}^{2}-2 B r}$ (Nye 1952).

Sample solutions are shown in figure 11 (cf. Osmond \& Griffiths, who solved (4.1) numerically). As $b \rightarrow h_{m}$, the domes are increasingly slumped ( $b \propto S^{-1}$, so a decrease in $b$ corresponds to an increase of the slope). The solution given above no longer works if $b<h_{m}$, and reflects the fact that the dome is unable to support itself against gravity. The limiting solution for $b \rightarrow h_{m}$ develops into a channel flow with semicircular cross-section, as illustrated in figure 11(e).

The next step in determining the history of an extrusion is to compute the dome volume from the characteristics solution:

$$
V=\frac{2 B h_{m}^{2}}{S^{3}} \int_{0}^{1} \frac{\mathrm{d} s}{s^{3} \sqrt{1-s^{2}}}\left[\left(3 \psi^{2}+s^{2}\right) \tan ^{-1}(s / \psi)-3 \psi s\right],
$$

where $\psi=\sqrt{b^{2} / h_{m}^{2}-1}$. The quantity $S^{2} V / b^{3}$ is plotted as a function of $h_{m} / b$ in figure $12(a)$. From this figure we observe that, as the volume increases during an extrusion $(V=Q t), h_{m} / b$ increases from zero up to a maximum value of unity. Thence, figure 11 can be regarded as a succession of snapshots of an evolving dome. Using the formulae in (4.7)-(4.8) and figure 12(a), we may further determine the time evolution of the edge of the slumped dome, and, in particular, its characteristic lengths, $X_{\min }, X_{\max }$ and $Y_{s}$. A sample extrusion constructed in this way is shown in figure $12(b-e)$ for a specific choice of $B$. From (4.11), we may also deduce the limits $V \sim\left(1-h_{m} / b\right)^{3 / 2}$ for $h_{m} \ll b$, and $V \sim \log \left(b / h_{m}-1\right)$ for $h_{m} \rightarrow b$. These, in turn, imply that

$$
h_{m} \sim t^{1 / 5}, \quad X_{\min } \sim t^{2 / 5}, \quad X_{\max } \sim t^{2 / 5}, \quad Y_{s} \sim t^{2 / 5} \quad \text { for } h_{m} \ll b
$$



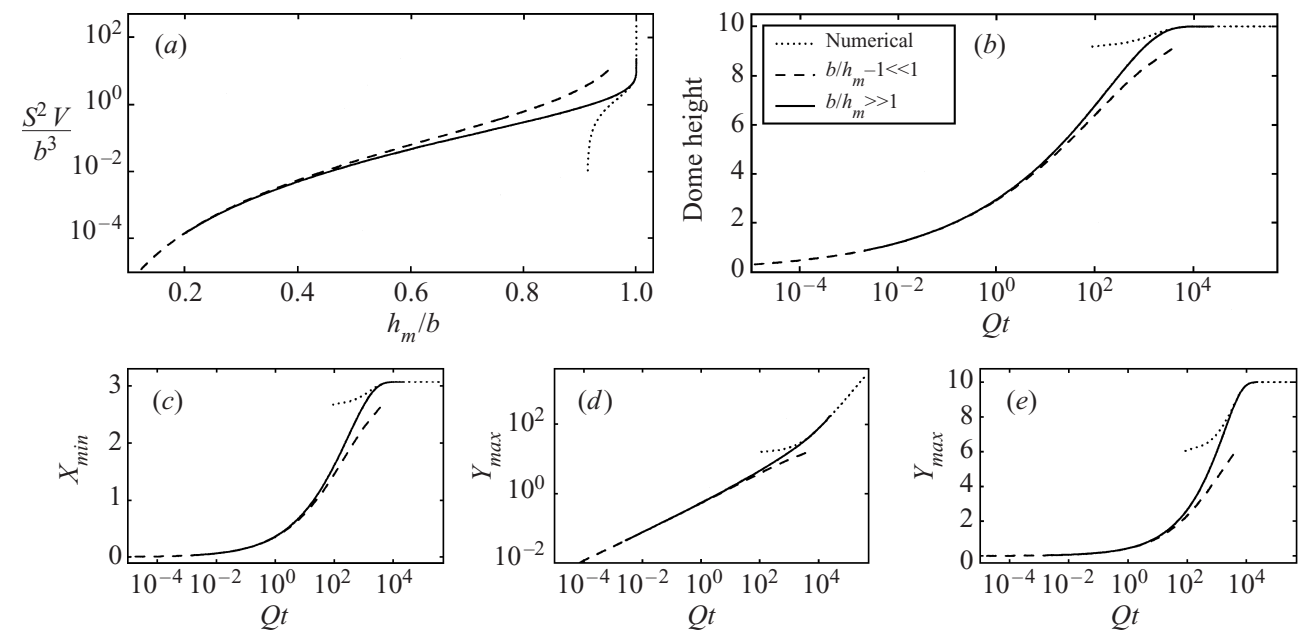

FIGURE 12. (a) $S^{2} V / b^{3}$ as a function of $h_{m} / b=S h_{m} / B$, computed by evaluating the quadrature in (4.11) numerically. For $b \gg h_{m}, S^{2} V / b^{3} \sim 2 \pi h_{m}^{3} /\left(15 \psi^{3} b^{2}\right)$, where $\psi^{2}=b^{2} / h_{m}^{2}-1$, whilst for $b / h_{m}-1 \ll 1, S^{2} V / b^{3} \sim \pi h_{m}^{3}[\log (2 / \psi)-3 / 2] / b^{2}$. These two limits are also included in the figure. Given the function shown in $(a)$, we may construct sample extrusions in the large- $B$ limit given specific values of $S$ and $B$. This is done in the other panels of the figure for $B=10, S=1$ : $(b)$ dome height, $(c)$ upstream length, $(d)$ downstream length, $(e)$ width. Again, the two limiting forms of $V$ are added in $(c-e)$.

and

$$
h_{m} \sim t^{0}, \quad X_{\min } \sim t^{0}, \quad X_{\max } \sim t^{1}, \quad Y_{s} \sim t^{0} \quad \text { for } h_{m} \rightarrow b,
$$

which correspond to the small- and long-time limits of an actual extrusion. The first limit mirrors the axisymmetrical expansion of a horizontal dome. The scalings are reported in table 1.

The final piece of the puzzle for evolving extrusions is to locate the small but finite yield surface and compute the depth-integrated velocity field. To accomplish this, we must return to the height evolution equation (2.4) which, in the large- $B$ limit and away from any localized vents, becomes

$$
h_{t}+\mathscr{U}_{x}+\mathscr{V}_{y}=0, \quad\left(\begin{array}{c}
\mathscr{U} \\
\mathscr{V}
\end{array}\right)=F\left(\begin{array}{c}
S-p \\
-q
\end{array}\right),
$$

where

$$
F=\frac{n B^{1 / n-1}}{n+1} Y^{1+1 / n} h^{2-1 / n}
$$

Equation (4.14), in fact, determines $Y$. To see this, we substitute the characteristics solution into the equation. The time dependence of that solution enters parametrically through $h_{m}(t)$, and, formally, we may write $h=h\left(x, y, h_{m}\right)$ and $a=a\left(x, y, h_{m}\right)$. However, given the implicit form of the solution is it simpler to make the coordinate transformation $(x, y) \rightarrow(a, h)$, and then write $x=x\left(a, h, h_{m}\right)$ and $y\left(a, h, h_{m}\right)$. The first result is that

$$
\left(\frac{\partial h}{\partial t}\right)_{x, y}=\frac{p(a, h)}{p\left(a, h_{m}\right)} h_{m t}
$$



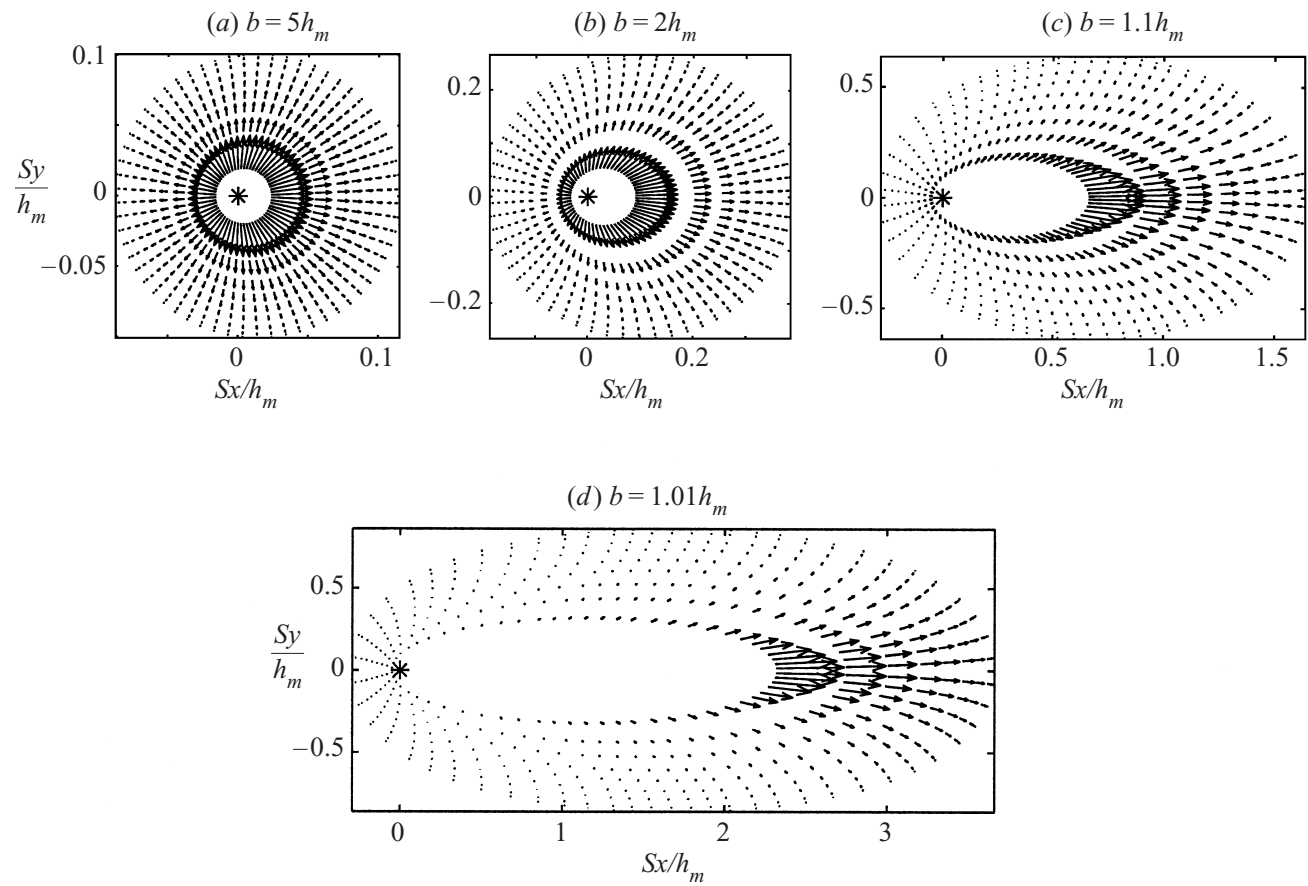

FIGURE 13. Pattern of the depth-averaged velocity field for the domes shown in figure $11(a-d)$.

Secondly, by writing $\mathscr{U}=F(S-p)$ and $\mathscr{V}=-p F / a$, we find that

$$
\mathscr{U}_{x}+\mathscr{V}_{y}=p\left[F\left(S-p-\frac{p}{a^{2}}\right)\right]_{h}-F\left[a_{x} p_{a}+a_{y}(p / a)_{a}\right],
$$

where $a_{x}=(\partial a / \partial x)_{h, t}, p_{a}=(\partial p / \partial a)_{h, t}$ and so on. After some algebra, we eventually find

$$
F\left(a, h, h_{m}\right)=\frac{a h h_{m t}}{S p\left(a, h_{m}\right)\left[\Gamma(a, h)-\Gamma\left(a, h_{m}\right)\right]}\left[\Gamma\left(a, h_{m}\right) \sin ^{-1}\left(\frac{h}{b \sqrt{1+a^{2}}}\right)-h\right],
$$

where $\Gamma(a, h)=\sqrt{b^{2}+a^{2} b^{2}-h^{2}}$. Finally, we compute the yield surface and the depthaveraged velocity field:

$$
Y=\left[\frac{n+1}{n} B^{(n-1) / n} h^{(1-2 n) / n} F\right]^{n /(n+1)}, \quad\left(\begin{array}{l}
\mathscr{U} \\
\mathscr{V}
\end{array}\right)=F\left(\begin{array}{c}
S-p \\
-p / a
\end{array}\right) .
$$

This reduces to $Y=\left[(n+1) h^{1 / n} h_{m} h_{m t}\left(3 h_{m}^{2}-h^{2}\right) /\left(6 n r B^{2+1 / n}\right)\right]^{n /(n+1)}$ and radial flow when $S \rightarrow 0$ (and completes the large- $B$ analysis of Balmforth et al. 2000).

Along the midsection, $a \rightarrow \pm \infty$, giving

$$
F \rightarrow \frac{h^{2} h_{m} h_{m t}\left(3 h_{m}^{2}-h^{2}\right)}{3 B\left(h_{m}^{2}-h^{2}\right)\left(B \mp S h_{m}\right)}, \quad\left(\begin{array}{c}
\mathscr{U} \\
\mathscr{V}
\end{array}\right)= \pm \frac{B F}{h}\left(\begin{array}{l}
1 \\
0
\end{array}\right) .
$$

The midsection profiles of $h$ and $Y$ constructed from (4.9) and (4.20) agree tolerably well with numerical computations like that shown in figure 4 . Note that $F$, and therefore the yield surface, diverges as $h \rightarrow h_{m}$. The beginnings of such a divergence near dome centre is visible in figure 4. However, in the numerical models, the vent has a finite size and any singularity is healed over the vent region, keeping the overall yield surface small. 


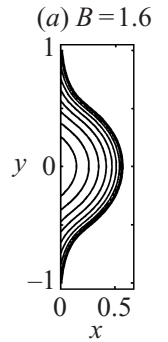

(d) $B=1.01$

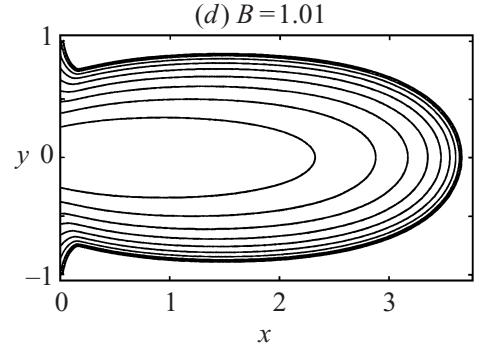

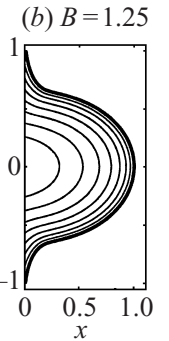

(c) $B=1.1$

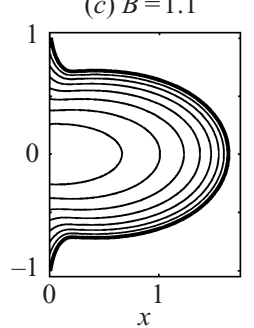

(e) Limiting shape

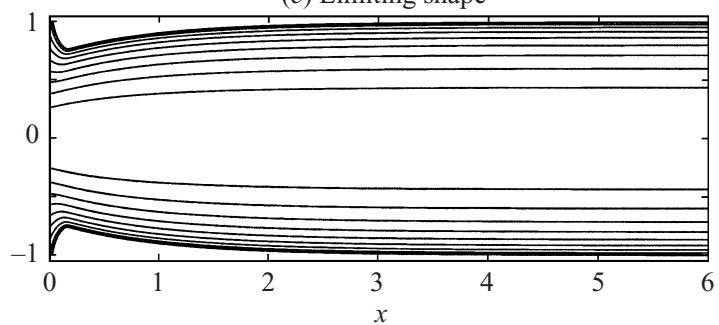

Figure 14. Contours of constant height (spaced by 0.1 ) for stationary rivulets emerged from a Gaussian orifice with half-width $y_{*}=0.8$. Shown are four values of $B$, and the limiting shape as $B \rightarrow 1$ (with $S=1$ ).

The patterns of the depth-averaged velocity fields, $h^{-1}(\mathscr{U}, \mathscr{V})$, for the sample domes of figure 11 are shown in figure 13. As the slope increases, the strongest speeds become confined to the segment of the dome downslope of the vent, and there is some similarity to a plug flow bordered by nearly motionless levees. However, the central velocity profile is never flat and the fluid always spreads laterally.

\subsection{The final stationary state of rivulets and slumps}

With the characteristics solution we now explicitly construct the final stationary shapes adopted by rivulets that brake to a halt, and slumps. For stationary rivulets emerged from an upstream aperture, instead of defining the solution by its apex, we prescribe the height field, $h(0, y)=h_{u}\left(y_{0}\right)$, along the uphill boundary, $x=0$ and $y=y_{0}$, as described in $\S 3.2$. Now, $a$ is a function of the parameterization of the boundary, $y_{0}$ :

$$
a=\frac{S h_{u}-\sqrt{B^{2}-h_{u}^{2}\left(h_{u}^{\prime}\right)^{2}}}{h_{u} h_{u}^{\prime}} .
$$

This statement of the boundary condition is valid provided $B>\left|h_{u} h_{u}^{\prime}\right|$. The implicit solution is now

$$
\begin{gathered}
S x=h-h_{u}-\frac{b}{2} \log \frac{\Delta(h)}{\Delta\left(h_{u}\right)}, \\
S\left(y-y_{0}\right)=\sqrt{b^{2}+a^{2} b^{2}-h^{2}}-\sqrt{b^{2}+a^{2} b^{2}-h_{u}^{2}} .
\end{gathered}
$$

For a Gaussian aperture, $h_{u}\left(y_{0}\right)=\exp \left[-\left(y_{0} / y_{*}\right)^{2}\right]$, with $y_{*}=0.8$, the outflow is shown for varying values of $b$ in figure 14. The limiting shape for $b=B / S \rightarrow 1$ is again an infinite stream with a semicircular profile far downstream. The profile of the midsection and the maximum downstream length for $B / S=1.1$ are compared with numerical solutions in figures 8 and 9 .

The boundary condition in (4.21) fails to provide the starting points for the characteristics when $B<\left|h_{u} h_{u}^{\prime}\right|$. This occurs because the structure can only be stationary 


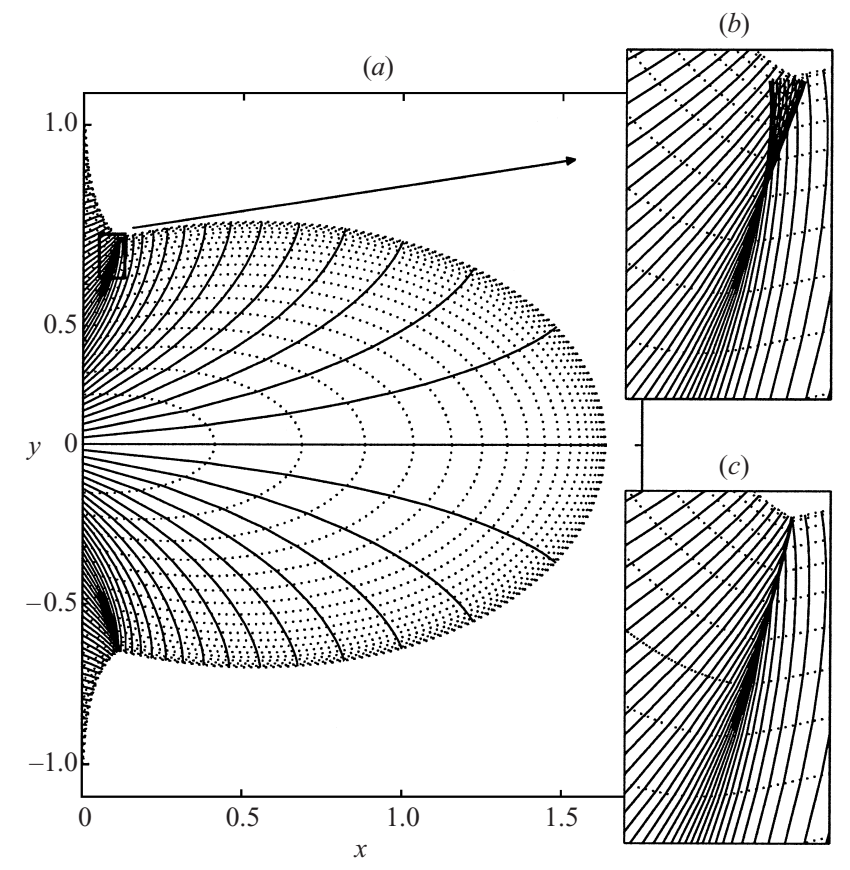

FIGURE 15. The characteristics solution for a rivulet emerging from a Gaussian aperture with $B=1.1$ and $y_{*}=0.66$. The region over which the characteristics cross is shown in more detail in $(b)$. Panel $(c)$ shows the single-valued surface that we construct by inserting a 'seam' into the solution according to the argument given in the main text.

provided $B \geqslant h \sqrt{h_{y}^{2}+\left(S-h_{x}\right)^{2}} \geqslant\left|h h_{y}\right|$. Hence, if $B<\left|h_{u} h_{u}^{\prime}\right|$, the fluid is unable to support itself against flow across the plane (in the $y$-direction) and the characteristics become complex. The limiting condition, $B=\max \left(\left|h_{u} h_{u}^{\prime}\right|\right)$, can be written in the form $B=B_{c}\left(y_{*}\right)$, which is also drawn in figure 7. For $B<B_{c}\left(y_{*}\right)$, the cross-slope slump of the fluid creates narrow boundary layers surrounding the aperture, as are visible in the numerical solution shown in figure 6.

Profiles like those shown in figure 14 are straightforward to construct provided the characteristics are simple curves that do not cross. Unfortunately, this does not always remain the case. For example, for the Gaussian aperture, even if $B>B_{c}\left(y_{*}\right)$, there are choices of $y_{*}$ for which the characteristics cross near the inlet (see figure 15). In this situation, the surface that one constructs for $h(x, y)$ is multi-valued and therefore unphysical (literally, the fluid structure contains voids).

Crossing characteristics are by no means unusual: similar problems invariably arise in constructing the final stationary states of slumps. In figure 16, we show the multivalued surface resulting on attempting to reconstruct one of the slumped structures of figure 10. In this case, the construction of the characteristics proceeds by fixing the fluid edge; i.e. we use (4.4)-(4.5) with $x_{0}$ and $y_{0}$ given by the computation. To prescribe $a$, we note that $h=h(x(a, \tau), y(a, \tau))$ implies $\partial h / \partial a=h_{x} \partial x / \partial a+h_{y} \partial y / \partial a$. But the edge is a curve of constant $h$ and $\tau$, and $h_{y}=a h_{x}$. Hence, $a=-(\partial y / \partial a) /(\partial x / \partial a) \equiv-\mathrm{d} y_{0} / \mathrm{d} x_{0}$ along this curve.

To remove the ambiguity in the multi-leaved surfaces, we must, in principle, return to the full height evolution equation. However, given the form of that equation, any discontinuity in the profile itself would probably force flow over the plane. Hence, we 


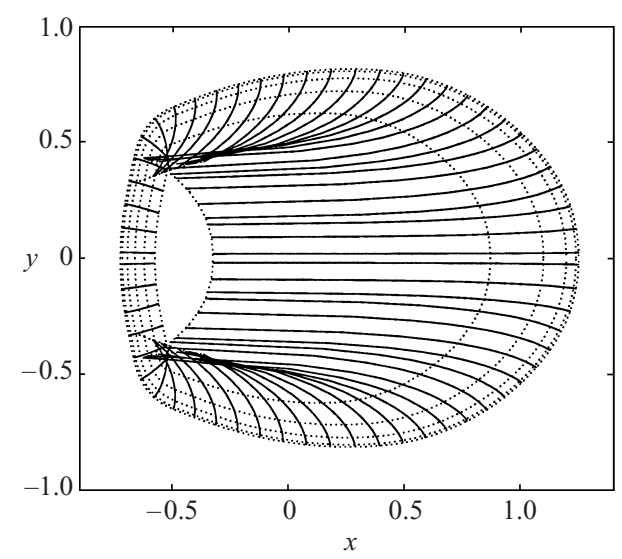

FIGURE 16. The characteristics solution for the slumped dome corresponding to the computation shown on the right of figure 10 , with $B=1 / 2$ and $\left(x_{*}, y_{*}\right)=(1 / 2,1 / 2)$. The solution is constructed using the dome edge at the end of the computation, and assumes that the structure yielded everywhere during the slump and has come to rest, neither of which is quite correct. However, the picture illustrates only how the characteristics cross. The characteristics are drawn only up to a certain height less than the maximal value for the sake of clarity (the crossings of the characteristics otherwise makes the picture unreadable).

argue that the only rule for rendering the solution single-valued which is consistent with a stationary profile is the one that leaves the surface continuous. This leads us to trace each characteristic from its starting point (in the aperture, or along the edge) up to the region in which the curves begin to cross. We then continue only as far as the point at which a second characteristic crosses the first one, with exactly the same value of $h$. At that stage, we switch to the second characteristic and proceed forward. This produces a single-valued surface with a 'seam' cutting through the old multi-valued region. The seam is a curve of discontinuous surface gradient, as illustrated in figure 15 for the stationary rivulet. (From a practical perspective, the construction of the seam is involved; some guidelines are offered in Appendix C.) We have not proved that the solutions to the thin-film equation actually limit to these constructions, but they do at least seem plausible. Moreover, the ridges in the numerical computations of figure 10 closely line up with the positions of the seams of the reconstructed characteristics solution, indicating that these features could well be the smoothed relatives of the seams. And once the seam is taken into account, the midsection profile of the computed slump agrees tolerably with that of the characteristics solution.

\section{Discussion}

In this article, we have considered the evolution of thin films of viscoplastic fluids on inclined planes. We specialized to relatively slow flows, which restricts the inclination of the slope to low values, and so we have not studied the larger Reynolds number flows that may be appropriate to some mud flows and submarine gravity currents. We also considered flow over uniform planes, but it is straightforward to extend the theory to uneven surfaces and channels (Mei \& Yuhi 2001). Our primary aim was to give a theoretical discussion of the problem explored experimentally by Coussot \& Proust (1996), Coussot et al. (1996) and Osmond \& Griffiths (2001), and lay the foundation for future work. This raises the question of how our results 
compare with the earlier experiments, and we conclude our study with some relevant remarks.

For domes extruded from a small vent, the most extensive and detailed experiments are those performed by Osmond \& Griffiths. These experiments used very low flow rates in order to access the regime of relatively strong yield stress. Hence, the analogous theory is that of large $B$, as given in $\S 4$. In fact, Osmond \& Griffiths complement their experiments with numerical solutions of the height-field equation (4.1), which is the basis of that theory, and show fair agreement between the two. The experimental data do not permit a more detailed comparison of either the velocity field or the asymptotic temporal scalings of the dome attributes, which our theory also provides.

Coussot \& Proust explored the structure of rivulets flowing from an upstream aperture. They focused on the shape of steady streams, and again provided a complementary theory based on a simplification of the thin-layer model. Here, we have been concerned with the transient dynamics of rivulets, notably the cases that brake to a halt, so we are unable to compare with their experiment.

Finally, Coussot et al. (1996) performed experiments in which they spilled a fixed volume of fluid onto an inclined plane and measured the shape of the final stationary deposit. This experiment permits a more detailed comparison with theoretical slumps. In fact, Coussot et al. already offer a comparison with approximate solutions of (4.1) which also dictates the shape of the final deposit. However, despite the partial agreement claimed in that article, there is a more significant disagreement which is not emphasized associated with the widths of the final structure: given that the fluid is initially spilled onto a localized area, one is tempted to compare the experiment with the theory for a slumped dome with a single pinnacle (as in §4.1). Notably, the maximum dimensionless width is unity. In all of Coussot's experiments, however, the width exceeds unity (as already pointed out by Osmond \& Griffiths).

In order to verify the apparent disagreement between the theory and the experiment, we performed our own 'spill tests' with a commercially available kaolin-based slurry ('joint compound' mixed with water). These experiments indicate that, indeed, there was a discrepancy, but also pointed to the explanation: the appropriate theoretical model is not an inclined dome with a single pinnacle, but one that possesses a sharp arc-shaped ridge, as we found in $\S 3.3$. Thus, the theory is not quite correct because the characteristic curves of the solution cross to produce a multi-valued and unphysical solution; the removal of the multi-valuedness inserts seams of discontinuous surface gradient into the solution in place of the pinnacle, as described in $\S 4.2$. Once one builds this feature into the solution, one can directly compare theory and experiment, which is the reason why Coussot et al.'s comparison of the thickness profiles was successful despite the discrepancy in the dimensionless widths.

In figure 17 we show one of our experiments and compare it directly to the theory. The theoretical structure has a pronounced seam which coincides with various surface features on the experimental dome, and there is agreement in the overall shape. A key feature of the seam is that it reduces the predictive power of the theory: if the dome had a single pinnacle, the slope and the shape of the fluid edge could be used to independently estimate the yield stress. However, if a seam occurs, the fluid edge defines only where it must lie, and a further ingredient, such as the maximal height, is required to compute the yield stress. In our opinion, the most useful attribute of the slump is the profile of the midsection, which automatically incorporates the maximal height, and furnishes a convenient set of measurements to compare theory and experiment. Such a comparison is shown in figure $17(b)$, and confirms the utility of the theory. 
(a)
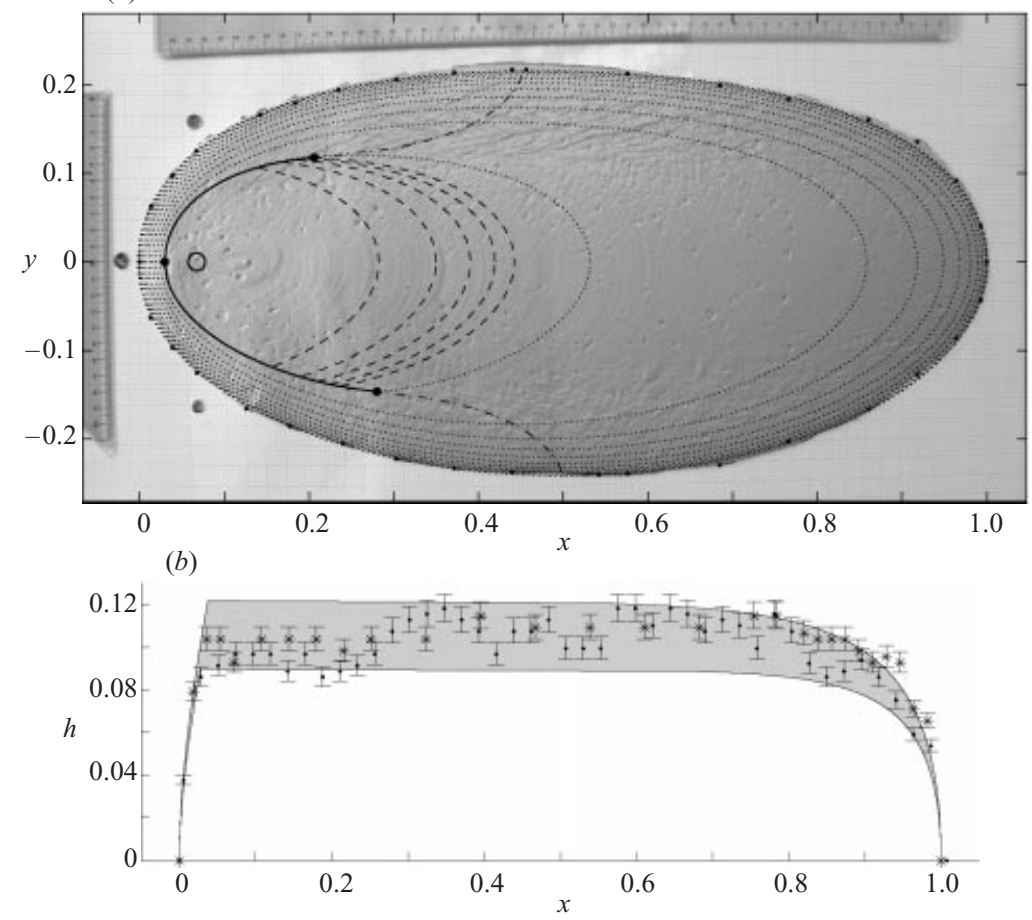

(c)

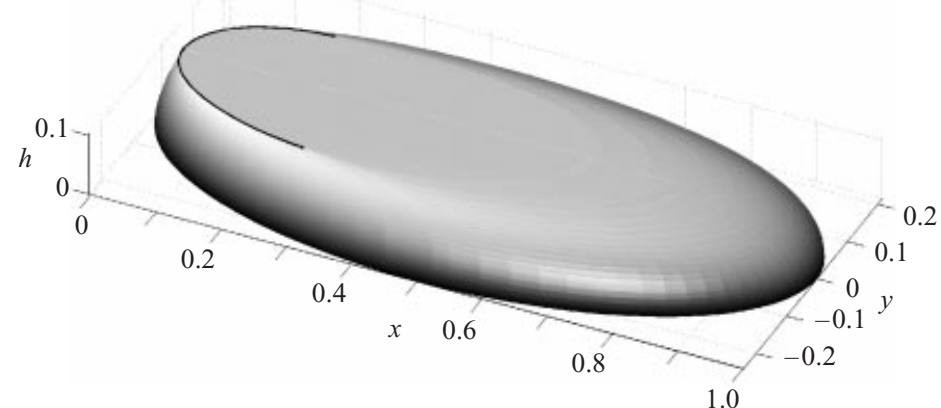

FiguRE 17. Comparison between the theory and an experiment in which we spilled a fixed volume (about 11) of a kaolin-based slurry onto an inclined plane. The slurry was formed by diluting 'joint compound' (Sheetrock Brand, United States Gypsum Company) with water (in the ratio of $7.5 \mathrm{~kg}$ of compound to $11 \mathrm{~kg}$ of water). (a) Theoretical contours of constant thickness superposed onto a photograph from the experiment. The dotted contour levels are spaced by 0.01 , whilst the dashed contours near the maximal height are spaced by $4 \times 10^{-5}$. The dashed-dotted line shows the characteristic along which the seam first occurs, and the solid curve delimited by dots is the seam. The dot midway along the seam is the position of maximum height. $(b)$ The theoretical and experimental midsection profiles. The characteristics solution is constructed from the observed fluid edge (fit with the function $y^{2}=f(x)$, where $f(x)$ is a quartic polynomial); the full solution, with its seam, is shown in $(c)$. The lengths and thickness are shown in dimensionless units obtained using the total slopewise length of the deposit, and $S=1$ (which fixes $H=L \tan \phi$ and not to be the maximum thickness). The slope angle is $\phi \approx 9.5^{\circ}$, and the slurry is spilled into the localized region indicated by the circle in $(a)$. The yield stress, $\tau_{p} \approx 22 \mathrm{~Pa}$ s, was estimated by spilling the slurry onto a horizontal plane (cf. Osmond \& Griffiths), and using the formula, $\tau_{p}=\rho g$ (height) ${ }^{2} /($ diameter) $\left(\rho \approx 1440 \mathrm{~kg} \mathrm{~m}^{-3}\right)$. From $\tau_{p}, L$ and $\phi$, we estimate that $B=\tau_{p} /\left(\rho g L^{2} \sin \phi\right) \approx 0.1$. Two sets of independent measurements of thickness are shown in $(b)$, and the spread of points gives some idea of the measurement error. The vertical error bars and the shaded theoretical band indicate the uncertainties stemming from the non-dimensionalization of $h$ (that is, $H$ ) and contained in $B$. 
We close by mentioning that our theory for yield-stress-dominated domes is closely connected to the classical theory of plasticity (Hill 1950). The main difference is that the 'plastic' region is a slab of fluid deforming under the action of stresses that are very slightly above the yield stress, and thin yielded boundary layers arise beside any walls. By contrast, standard plasticity theory often assumes a boundary condition based upon Prandtl's friction law (wall shear stress is proportional to the yield stress) to allow the plastic material to slip over the bounding walls; the constant of proportionality, the friction factor, becomes a parameter in the problem (Sherwood \& Durban 1998).

The financial support of an EPSRC Advanced Fellowship is gratefully acknowledged by R. V.C. This work was supported by the National Science Foundation (Grant No. DMS 72521).

\section{Appendix A. Spreading uniform rivulets}

In this and the following Appendix we retreat to two simpler problems: a uniform rivulet directed downhill (so $\partial_{x}=0$ ), and a line source on a plane (with $\partial_{y}=0$ ). An expanding horizontal dome can also be dealt with in a straightforward fashion (Balmforth et al. 2000). We deal with the rivulet first.

The thin-layer theory is encapsulated in the equations,

$$
h_{t}=\partial_{y}\left[\frac{n \operatorname{sgn}\left(h_{y}\right)}{n+1}\left|h_{y}\right|^{1 / n} Y^{1+1 / n}\left(h-\frac{n Y}{2 n+1}\right)\right], \quad Y=\operatorname{Max}\left(h-\frac{B}{\sqrt{S^{2}+h_{y}^{2}}}, 0\right) \text {. }
$$

There is no mass source, and the fluid flows from an initial profile given by $h(y, t=0)$ $=\exp \left[-\left(y / y_{*}\right)^{2}\right]$. Provided $Y>0$, the rivulet flows downslope, and decelerates as a whole once it spreads.

Two sample spreading rivulets with different yield stresses are shown in figure 18 . For the larger value of $B$, the fluid slumps sideways to an equilibrium characterized largely by $Y=0$. That state is given by

$$
h=\sqrt{b^{2}-\left(S y+\sqrt{b^{2}-h_{m}^{2}}\right)^{2}},
$$

where $h_{m}=h(0, t \gg 1)$ is the final maximal height $(b=B / S)$. In this formula, $b$ must exceed $h_{m}$, which sets an upper bound on the cross-sectional area of a stationary rivulet. Provided the bound is larger than the cross-sectional area of the initial rivulet (as given by the initial width $y_{*}$ ), the fluid can relax to a stationary rivulet with $h_{m}<b$. If, however, the initial width is any larger, there is more fluid than can be locked into a stationary rivulet and the fluid will spread continually. The computation with lower $b$ in figure 18 illustrates this second situation.

\section{Appendix B. Line sources}

For line sources (sheet flow),

$$
h_{t}+\partial_{x}\left[\frac{n \sigma}{n+1}\left|S-h_{x}\right|^{1 / n} Y^{1+1 / n}\left(h-\frac{n Y}{2 n+1}\right)\right]=w_{s}, \quad Y=\operatorname{Max}\left(h-\frac{B}{\left|S-h_{x}\right|}, 0\right)
$$



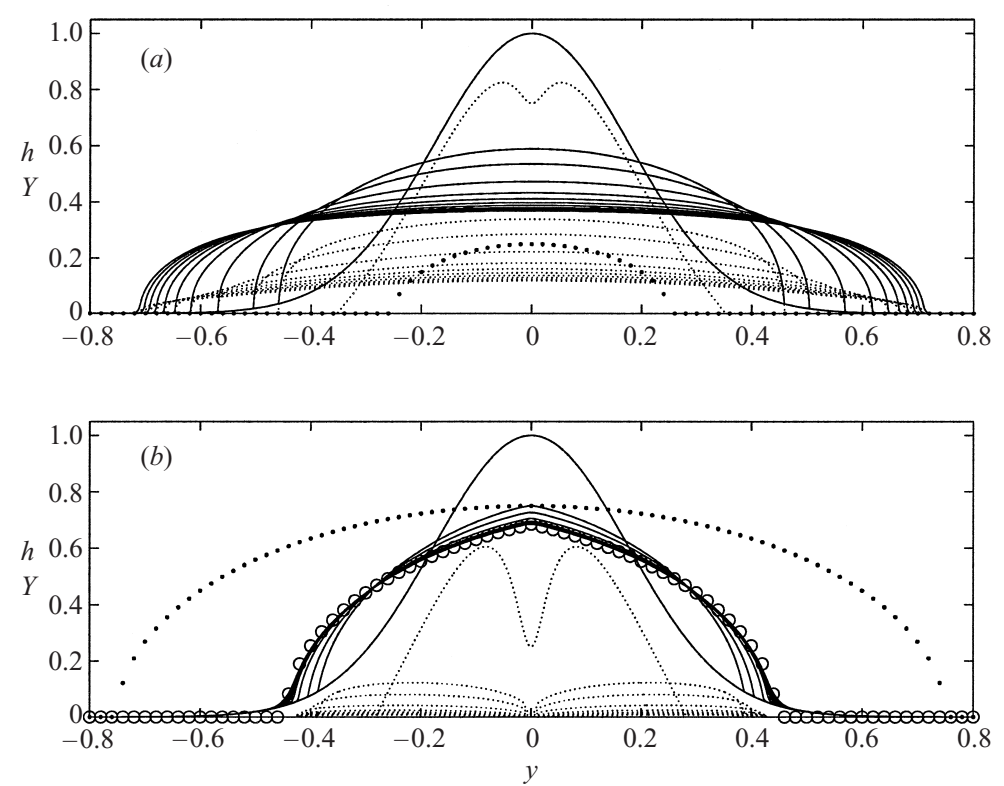

FIGURE 18. The thickness (solid lines) and yield surfaces (dotted lines) for spreading uniform rivulets with $(a) B=1 / 4$ and $(b) B=3 / 4$. The initial profile has a characteristic half-width of $y_{*}=0.25$. The dots show the maximum stationary profile at that yield stress; the circles in $(b)$ show the approximate final shape. The solutions are shown at intervals of 1.25 in $t$.

and

$$
\frac{\mathrm{d}}{\mathrm{d} t} \int_{X_{\max }}^{X_{\min }} h(x, t) \mathrm{d} x=Q,
$$

where $\sigma=\operatorname{sgn}\left(S-h_{x}\right)$, and $X_{\max }$ and $X_{\min }$ denote the edges of the flow.

Sample evolving flows are shown in figures 19 and 20 . The source function used is a one-dimensional analogue of (3.1): $w_{s}=50\left[\operatorname{Max}\left(1-2500 x^{2}, 0\right)\right]^{2}$ (giving $Q=16 / 15$ ). Because of their relative simplicity, these one-dimensional examples can be used to verify in detail the similarity scalings and $B \gg 1$ asymptotic theory.

\section{B.1. Similarity scalings}

We look for similarity solutions by considering infinitely thin sources and setting $x=\xi t^{\gamma}$ and $h(x, t)=\varphi(\xi) t^{1-\gamma}$. The evolution equation then becomes

$$
\varphi-\gamma(\xi \varphi)_{\xi}+t^{(2+1 / n)(1-\gamma)}\left[\frac{n \sigma}{n+1}\left|S-t^{1-2 \gamma} \varphi_{\xi}\right|^{1 / n} \mathscr{Y}^{1+1 / n}\left(\varphi-\frac{n \mathscr{Y}}{2 n+1}\right)\right]_{\xi}=0,
$$

where

$$
\mathscr{Y}=\varphi-\frac{B t^{\gamma-1}}{S-t^{1-2 \gamma} \varphi_{\xi}}, \quad Q=\int_{-\infty}^{\infty} \varphi \mathrm{d} \xi
$$

and $\sigma=\operatorname{sgn}\left(S-t^{1-2 \gamma} \phi_{\xi}\right)$.

The small-time similarity solution is given by $\gamma=2(n+1) /(2 n+3)$ and is identical to that for an extrusion of a power-law fluid onto a flat plane. For $n=1$, the maximal thickness and fluid edges scale as

$$
h_{m} \sim t^{1 / 5}, \quad X_{\max }=-X_{\min } \sim t^{4 / 5} .
$$

All other terms in (B 3) and (B 4) are sub-dominant for $t^{-1} \rightarrow \infty$, but not as time 

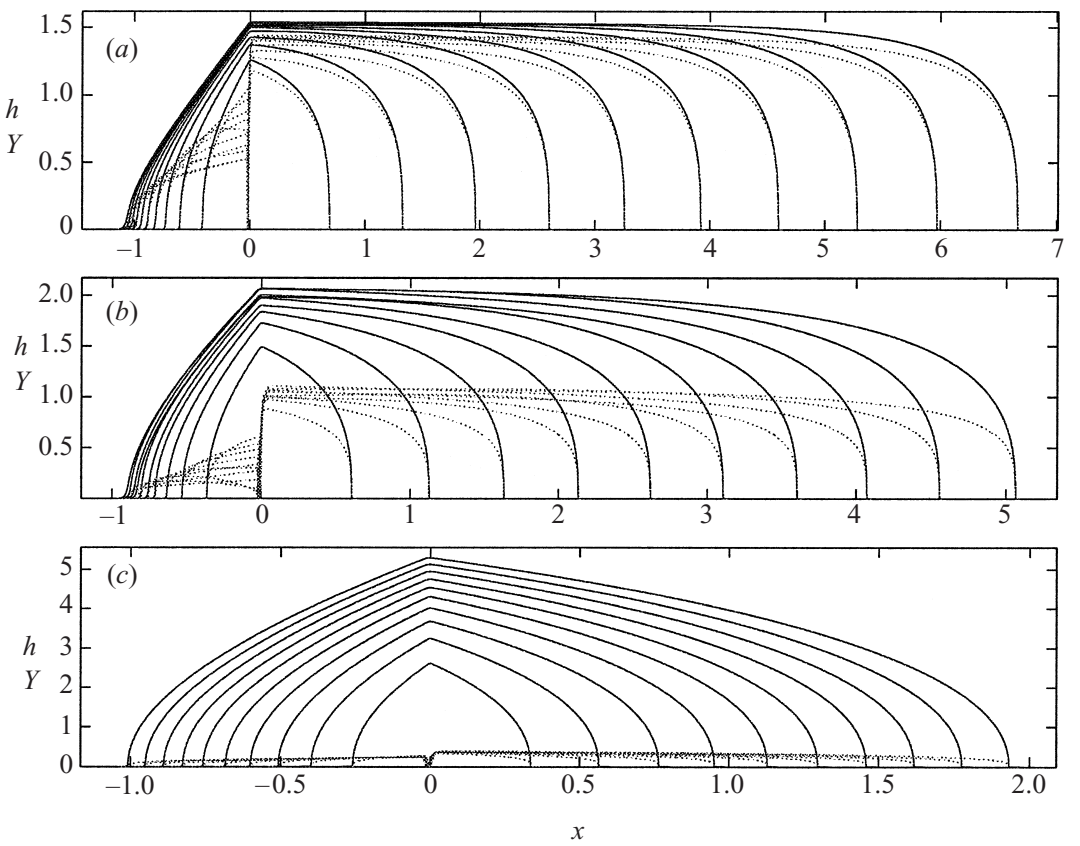

FiguRE 19. The thickness and yield surfaces for constant extrusion from a line source with (a) $B=0.1$, (b) $B=1$ and (c) $B=10$ (and $n=1$ ). The solutions are shown at unit intervals in $t$.
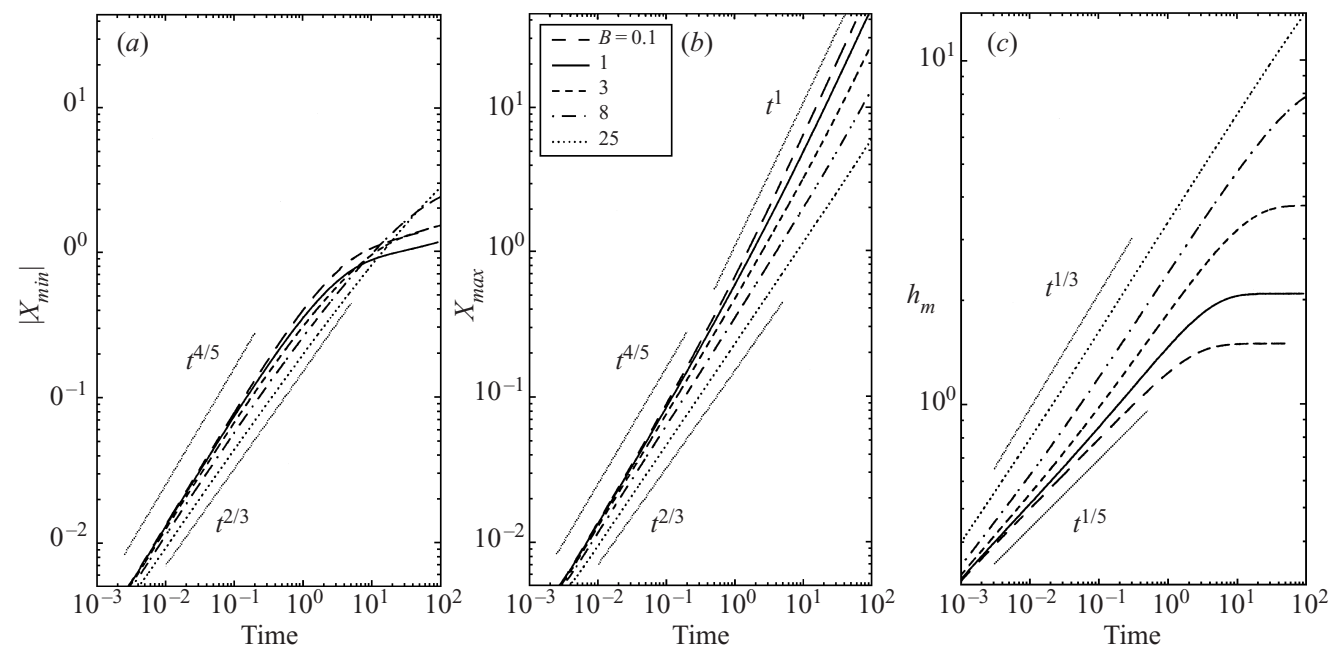

FiguRE 20. Evolution of $(a)$ upstream length, $(b)$ downstream length and $(c)$ maximum height for extrusions with varying $B$. For these computations, in order to better resolve the solution, the domain of the computation was split into upstream and downstream sections that moved approximately with the fluid edges, and the source function was replaced by a boundary condition at $x=0$.

proceeds forward. For the latter, the approximation breaks down in two possible ways: one is due to the slope-dependent terms in the combination, $S-t^{1-2 \gamma}$; the other is due to the yield-stress term in $\mathscr{Y}$. The slope-dependent terms become important for $t=O(1)$. The yield stress, on the other hand, can break the early-time scalings when $t \sim B^{1 /(2-3 \gamma)}$. Thus we anticipate two transitions from the early-time scalings 

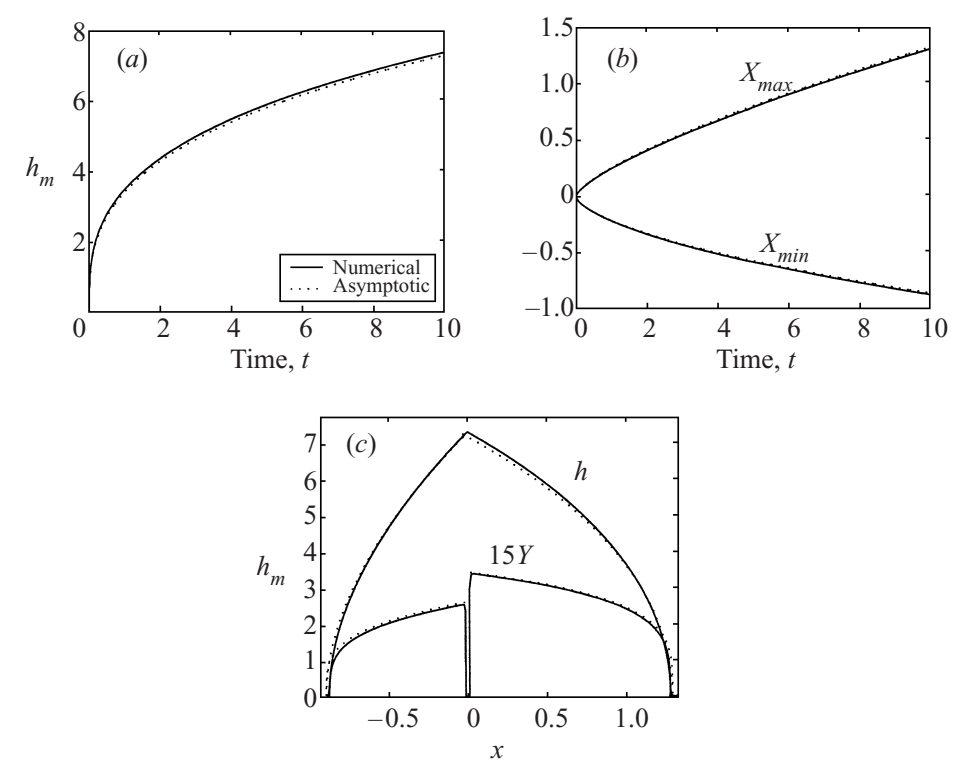

FiguRE 21. Comparison of the numerical and asymptotic solution for $n=1$ and $B=25:(a)$ maximal height, $(b)$ edge locations, $(c)$ thickness and yield stress. In $(c)$ the comparison is made at $t=10$.

that depend on the yield stress. For $B \ll 1$, the extrusion enters a slope-dominated regime given by $\gamma=1$,

$$
\xi \varphi_{\xi}=\left[\frac{n \sigma S}{n+1} \mathscr{Y}^{1+1 / n}\left(\varphi-\frac{n \mathscr{Y}}{2 n+1}\right)\right]_{\xi}
$$

and $\mathscr{Y}=\varphi-B / S$. The relevant solution is $\varphi=$ constant, which describes a uniform slab moving downslope (and must be corrected in a boundary layer at the fluid edge; cf. Lister 1992). As for a point source, the uphill part of the solution is not given by this relation, but instead comes to rest. The long-time scalings are now

$$
h_{m}, X_{\min } \sim t^{0}, \quad X_{\max } \sim t .
$$

The second transition, occurring for $B \gg 1$, is to a yield-stress-dominated state.

\section{B.2. Yield-stress-dominated situations}

When $B$ is large, $Y \rightarrow 0$. This limit can be explored using a formal expansion ( $h=B \mathscr{H}$, $x=B \tilde{x}, t=B^{2} \tilde{t}$ and $\left.Y=B^{-n /(n+1)} \tilde{Y}\right)$, but because we need only the leading-order terms, we show the analysis in terms of the original variables. For an infinitely thin line source, the equations become:

$$
\left(S-h_{x}\right) h= \pm B, \quad h_{t}=-\partial_{x}\left(\frac{n \sigma h}{n+1}\left|S-h_{x}\right|^{1 / n} Y^{1+1 / n}\right)
$$

and

$$
\frac{\mathrm{d}}{\mathrm{d} t} \int_{X_{\min }}^{X_{\max }} h(x, t) \mathrm{d} x=Q .
$$

We immediately find the solution for the thickness:

$$
h-h_{m} \pm b \log \frac{(b \mp h)}{\left(b \mp h_{m}\right)}=S x,
$$


where $h=h_{m}$ and $x=0$ and $b=B / S$. The edges of the fluid are given by the further relations,

$$
S X_{\max }=-h_{m}-b \log \left(1-h_{m} / b\right), \quad S X_{\min }=-h_{m}+b \log \left(1+h_{m} / b\right) .
$$

We substitute the solution into the conservation law to find

$$
S Q t=b^{2} \log \left(\frac{b+h_{m}}{b-h_{m}}\right)-2 b h_{m}
$$

which is in agreement with numerical computations (see figure 21).

Next consider the height evolution equation:

$$
h_{t} \pm \frac{n B^{1 / n}}{n+1}\left(h^{1-1 / n} Y^{1+1 / n}\right)_{x}=0,
$$

giving

$$
Y=\left[\frac{(n+1) h^{1 / n} h_{m} h_{m t}}{n S B^{1 / n}\left(b \mp h_{m}\right)}\right]^{n /(n+1)} .
$$

The two versions of this relation provide the fake yield surfaces upstream and downstream of the fluid apex (shown in figure 21c).

Finally, we may read off the temporal scalings:

$$
t \ll 1: \quad h_{m} \sim t^{1 / 3}, \quad X_{\max }=-X_{\min } \sim t^{2 / 3},
$$

and

$$
t \gg 1: \quad h_{m}, X_{\min } \sim t^{0}, \quad X_{\max } \sim t .
$$

All the various scalings are verified by the computations shown in figure 20 .

\section{Appendix C. Construction of the seam}

In $\S \S 4$ and 5 , we build solutions to the characteristic equations for stationary deposits that contain seams of discontinuous surface gradient and render the solution single-valued. In this Appendix, we offer some guidelines for how one performs such constructions.

We begin with the stationary rivulet, for which the characteristics cross near the aperture, see figure 15. First, we locate the values of $h$ for which crossings can potentially occur. The range begins at $h=0$, since the seam extends to the fluid edge. To find the upper limit of the range, $h_{c}$, we note that, at fixed height, $x$ should be a monotonic function of $y_{0}$, unless there are characteristics crossing. Hence, $h_{c}$ is the largest value of height for which $\mathrm{d} x / \mathrm{d} y_{0}=0$.

Next, we explicitly locate the seam: here, there are two characteristics with different values for $y_{0}$, say $y_{0 \alpha}$ and $y_{0 \beta}$, that must have the same position, $(x, y)$, and height, $h$. In other words,

$$
x\left(y_{0 \alpha}, h\right)=x\left(y_{0 \beta}, h\right), \quad y\left(y_{0 \alpha}, h\right)=y\left(y_{0 \beta}, h\right),
$$

where $x\left(y_{0}, h\right)$ and $y\left(y_{0}, h\right)$ are the two functions in (4.23). These equations can be solved for the labels of the characteristics, $y_{0 \alpha}$ and $y_{0 \beta}$, given $h$. In particular, by putting $h=0$ in $(\mathrm{C} 1)$, we determine the range of characteristics, $\left[y_{c \alpha}, y_{c \beta}\right]$, for which there are crossings.

Now we have all the information needed to locate the region over which the characteristics cross on both the $\left(y_{0}, h\right)$-plane and in real space $(x, y)$. To produce the 
surface, $h(x, y)$, we need only keep track of this special region, and monitor, using (C 1), when we must switch characteristics.

For the stationary slumps, the prescription is not very different. Now, the range of awkward height values is $\left[h_{c}, h_{m}\right]$, where $h_{m}$ is the maximum dimensionless height. The discriminant that we may use to detect crossings is $\mathrm{d} x / \mathrm{d} a$, on using $a$ to label the characteristics (assuming this to be unambiguous). We then compute $h_{c}$ and the location of the seam as above, but using (4.4)-(4.5) for $x(a, h)$ and $y(a, h)$. In this case, every characteristic crosses at least one other, because these curves all begin along the edge and then rise to increasing heights, ending only when they intersect a seam.

\section{REFERENCES}

Balmforth, N. J., Burbidge, A. S., Craster, R. V., Salzig, J. \& Shen, A. 2000 Visco-plastic models of isothermal lava domes. J. Fluid Mech. 403, 37-65.

BALmforth, N. J. \& Craster, R. V. 1999 A consistent thin-layer theory for Bingham fluids. J. Non-Newtonian Fluid Mech. 84, 65-81

Blom, J. G., Trompert, R. A. \& Verwer, J. G. 1996 Algorithm 758: VLUGR2: A vectorizable adaptive-grid solver for PDEs in 2D. ACM Trans. Math. Software 22, 302-328.

Coussot, P. 1997 Mudflow Rheology and Dynamics. IAHR Monograph Series, Balkema.

Coussot, P. \& Proust, S. 1996 Slow, unconfined spreading of a mudflow. J. Geophys. Res. 101, 25217-25229.

Coussot, P., Proust, S. \& Ancey, C. 1996 Rheological interpretation of deposits of yield stress fluids. J. Non-Newtonian Fluid Mech. 66, 55-70.

De Kee, D., Chabra, R. P., Powley, M. B. \& Roy, S. 1990 Flow of viscoplastic fluids on an inclined plane: Evaluation of yield stress. Chem. Engng Commun. 96, 229-239.

Griffiths, R. W. 2000 The dynamics of lava flows. Annu. Rev. Fluid Mech. 32, 477-518.

Hill, R. 1950 The Mathematical Theory of Plasticity. Oxford University Press.

HuANG, X. \& Garcia, M. H. 1998 A Herschel-Bulkley model for mudflow down a slope. J. Fluid Mech. 374, 305-333.

Hulme, G. 1974 The interpretation of lava flow morphology. Geophys. J. R. Astron. Soc. 39, 361-383.

HupPERT, H. E. 1982 The propagation of two-dimensional and axisymmetric viscous gravity currents over a rigid horizontal surface. J. Fluid Mech. 121, 43-58.

HutTer, K. 1983 Theoretical Glaciology. D. Reidel.

Lister, J. R. 1992 Viscous flows down an inclined plane from point and line sources. J. Fluid Mech. 242, 631-653.

LiU, K. F. \& MeI, C. C. 1989 Slow spreading of Bingham fluid on an inclined plane. J. Fluid Mech. 207, 505-529.

Mei, C. C. \& Yuni, M. 2001 Slow flow of a Bingham fluid in a shallow channel of finite width. J. Fluid Mech. 431, 135-159.

NYE, J. F. 1952 The mechanics of glacier flow. J. Glaciol. 2, 82-93.

Osmond, D. I. \& GRIFFITHS, R. W. 2001 The static shape of yield-strength fluids slowly emplaced on slopes. J. Geophys. Res. 106(B8), 16241-16250.

Sherwood, J. D. \& Durban, D. 1998 Squeeze flow of a Herschel-Bulkley fluid. J. Non-Newtonian Fluid Mech. 77, 115-121.

Sneddon, I. N. 1957 Elements of Partial Differential Equations. McGraw-Hill.

TANNER, R. I. 1985 Engineering Rheology. Clarendon.

Walton, I. C. \& Bittleston, S. H. 1991 The axial flow of a Bingham plastic in a narrow eccentric annulus. J. Fluid Mech. 222, 39-60.

Whipple, K. X. 1997 Open-channel flow of Bingham fluids: applications in debris-flow research. J. Geol. 105, 243-262. 